\title{
L'art perdu de " faire des chansons " : la chanson de composition locale dans la région du Détroit
}

\section{Marcel Bénéteau}

Volume 10, 2014

URI : https://id.erudit.org/iderudit/1039294ar

DOI : https://doi.org/10.7202/1039294ar

Aller au sommaire du numéro

\section{Éditeur(s)}

Société Charlevoix

Presses de l’Université d'Ottawa

ISSN

1203-4371 (imprimé)

2371-6878 (numérique)

Découvrir la revue

Citer cet article

Bénéteau, M. (2014). L’art perdu de "faire des chansons " : la chanson de composition locale dans la région du Détroit. Cahiers Charlevoix, 10, 255-314. https://doi.org/10.7202/1039294ar
Résumé de l'article

L'ethnologue Marcel Bénéteau s'intéresse à la chanson de composition locale, un secteur négligé dans le domaine de la chanson folklorique. Le plus souvent créées par des non-professionnels sur des airs connus, les chansons de ce type ont longtemps été considérées par les experts comme étant de facture maladroite, de contenu douteux et de valeur insignifiante comparées aux pièces importées de la France à l'époque coloniale. Conséquemment, les grands folkloristes du Canada français les ont peu recueillies. Pourtant, la facture de telles chansons, qui commémorent des événements locaux, qui mettent en valeur des incidents de la petite histoire et qui commentent les conditions sociales dans les communautés en question, est au coeur même de la démarche folklorique. « Faire des chansons » est une pratique qui engage les gens dans un dialogue avec la tradition et les amène à situer leur vécu dans le contexte plus large d'un procédé transmis d'une génération à l'autre. À partir d'un corpus de chansons locales composées dans la région de Windsor, l'auteur analyse les modalités de cet art de « faire des chansons ", depuis l'appropriation et l'adaptation de chants existants jusqu'à la création de nouvelles chansons. De plus, il s'intéresse à la fonction sociale des chansons locales ainsi qu'à leur apport aux études ethnologiques, linguistiques et historiques.
Ce document est protégé par la loi sur le droit d'auteur. L'utilisation des services d’Érudit (y compris la reproduction) est assujettie à sa politique d'utilisation que vous pouvez consulter en ligne.

https://apropos.erudit.org/fr/usagers/politique-dutilisation/ 
L'art perdu de « faire des chansons » :

la chanson de composition locale dans la région du Détroit

\author{
Marcel BéNéteau \\ Département de folklore et ethnologie \\ Université de Sudbury
}




\section{SOMMAIRE}

I - UNE FORME NÉGLIGÉE SUR UN TERRAIN MAL CONNU 257

$\begin{array}{lll}\text { II }- & \text { FAÇONS DE FAIRE DES CHANSONS } & 267\end{array}$

III - $\quad$ LA COMMUNAUtÉ DU DÉTROIT VUE PAR LES CHANSONS

ANNEXE

295 


\section{L'art perdu de « faire des chansons » : la chanson de composition locale dans la région du Détroit}

\section{I - UNE FORME NÉGLIGÉE SUR UN TERRAIN MAL CONNU \\ La chanson de composition locale}

Les folkloristes du Canada français ont démontré peu d'intérêt pour les chansons de composition locale. Les préjugés contre la forme sont de longue date. En 1919, Marius Barbeau écrivait :

Il faut établir une distinction entre les pièces qui viennent de France et celles - relativement peu nombreuses - qui furent composées au Canada [...] Tandis que la plupart des chants venus de France prirent naissance à une époque où les jongleurs (chanteurs ou compositeurs populaires) possédaient encore une éducation artistique particulière, les compositions canadiennes, elles, sortirent surtout de la classe des illettrés, ce qui explique sans doute la différence assez sensible que l'on remarque dans leur valeur littéraire ${ }^{1}$.

Plus tard, il proposera que « [c]es manifestations d'art rustique, tout intéressantes qu'elles puissent être, sont dépourvues de style autant que de grammaire. » Il cite à l'appui un collaborateur, l'abbé Pierre-Paul Arsenault, qui lui avait fourni de nombreuses pièces : « Il va sans dire que plusieurs de ces chansons feraient peut-être mieux de mourir dans $1^{\prime}$ oubli $^{2} »$. Carmen Roy n'est guère plus élogieuse sur cette partie du répertoire :

Dans l'ensemble, on peut dire que la chanson née au Canada, et retenue par la tradition, est une chanson d'origine populaire, en général assez gauche, composée par des chanteurs de village,

1. Marius Barbeau, «Chants populaires du Canada, recueillis parÉ.-Z. Massicotte et préparés par C-M. Barbeau », Journal of American Folklore, vol. XXXII, n CXXIII, janvier-mars 1919, p. .

2. Marius Barbeau, Romancero du Canada, Montréal, Les Éditions Beauchemin, 1937, p. 184. 
sur un air déjà existant et très connu. Des copies manuscrites de chansons circulent et, pour cette raison, elles ne « folklorisent» guère ${ }^{3}$.

En Ontario, Germain Lemieux en a à peine enregistré, préférant, comme ses maîtres, cataloguer l'héritage des anciennes chansons françaises sur le territoire nord-américain. On pourrait conclure à partir de son œuvre monumentale que ce genre de chanson n'a guère existé en Ontario français. Pourtant, le classement de la vaste collection de chansons recueillies par les étudiants du département de folklore de l'Université de Sudbury depuis 1980 relève un grand nombre de chansons de composition locale, attestant de la vitalité de la forme dans le nord ontarien. Il en est de même dans plusieurs autres régions du Canada, notamment en Acadie.

En dépit de leurs supposées insuffisances poétiques et musicales, ces objets culturels nous fournissent une occasion parfaite d'observer le processus dynamique d'une communauté en interaction avec la tradition. On peut les considérer comme éléments d'un " ethnotexte », concept développé au CNRS par Jean-Claude Bouvier et ses collaborateurs pour décrire l'ensemble des traditions orales reçues, façonnées et transmises par une communauté ${ }^{4}$. L'ethnotexte comprend les chansons, les contes, les proverbes, les légendes et les récits de vie, ainsi que le parler régional qui les véhicule; ce métatexte comporte effectivement ce que Bouvier appelle « le discours oral global qu'une communauté - rurale ou urbaine - tient sur elle-même, son passé, son présent ${ }^{5}$ ». Je proposerais que la chanson locale occupe une place privilégiée dans ce discours, donnant aux membres de la communauté un mécanisme qui leur permet de commenter directement les réalités de leur milieu en leur fournissant un moyen de réduire certains

3. Carmen Roy, Littérature orale en Gaspésie. Seconde édition revue et augmentée, Ottawa, Les Éditions Léméac Inc., 1981, p. 293-294.

4. Jean-Claude Bouvier, Henry-Paul Bremondy, Philippe Joutard, Guy Mathieu, Jean-Noël Pelen, Tradition orale et identité culturelle, problèmes et méthodologie, Paris, Éditions du Centre national de la recherche scientifique, 1980, p. i.

5. Ibid., p. i. 
écarts entre l'identité locale et l'appartenance culturelle.

Malgré la vitalité de la chanson locale ailleurs en Amérique française, mes propres enquêtes dans le sud-ouest de l'Ontario suggèrent que la tradition de " faire des chansons 》 est à toute fin pratique éteinte chez les francophones de cette région. Mais certaines sources historiques indiquent que ce ne fut pas toujours le cas ; des documents manuscrits et imprimés des XIX et $\mathrm{XX}^{\mathrm{e}}$ siècles révèlent que la pratique de composer des chansons au sujet de personnes et d'événements dans la communauté a longtemps eu cours dans la plus ancienne colonie française de l'Ontario. C'est en suivant ces pistes que je vais tenter de recréer ce répertoire et de développer une analyse de la forme et de la signification des chansons de composition locale.

Le présent article se propose de définir cette catégorie de chansons et de la situer dans le contexte général des études sur la chanson de tradition orale et, plus spécifiquement, de déterminer la place qu'elle occupe dans le répertoire du sud-ouest ontarien. Je vais ensuite examiner la forme et le contenu des chansons locales ainsi que le rôle qu'elles jouent dans une communauté. L'analyse portera sur une vingtaine de chansons recueillies sur le terrain ou tirées de sources manuscrites ou imprimées. En guise de conclusion, j'offrirai quelques commentaires sur ce qui semble être la disparition imminente de cette ancienne pratique sur le terrain du Détroit : pourquoi est-elle en train de disparaître? Pourquoi les gens arrêtent-ils de « faire des chansons »? Qu'est-ce qui est perdu avec la mort de cette tradition ? Qu'est-ce qui la remplace?

\section{Le terrain d'enquête}

Avant d'aborder le sujet, il convient de situer le terrain en question. Notre champ d'enquête se situe dans les communautés de la rive canadienne de la rivière Détroit et du lac Sainte-Claire (bien que certains des incidents décrits dans les chansons aient eu lieu du côté états-unien de la frontière). L'ancienne colonie du Détroit, fondée par les Français en 1701, comprenait autrefois les terres 
des deux côtés de la voie maritime. Le fait français disparut graduellement du côté des États-Unis tout au cours du XIX ${ }^{\mathrm{e}}$ siècle, mais la langue persiste toujours dans plusieurs communautés sur la rive canadienne du Détroit. Ce peuplement francophone est le plus ancien en Ontario et est resté jusqu'à tout récemment plus ou moins isolé du reste de la francophonie nord-américaine. La population originale fut renforcée par l'arrivée dans la deuxième moitié du XIX ${ }^{\mathrm{e}}$ siècle d'une deuxième vague d'immigration de la vallée du Saint-Laurent qui s'établit à l'est du premier groupe, sur les bords du lac Sainte-Claire. J'ai analysé ailleurs les différences culturelles et linguistiques qui caractérisent ces deux peuplements $^{6}$; dans la présente étude, je vais considérer dans son ensemble un répertoire qui comprend les traditions de ces les deux zones de peuplement.

L'ancienneté du peuplement, sa proximité de la frontière états-unienne et la distance qui sépare le Détroit des autres centres francophones de l'Ontario sont des facteurs incontournables dans le caractère et l'identité des francophones du Sud-Ouest affectant toute une variété d'éléments depuis la langue parlée, les références culturelles évoquées et les sentiments d'appartenance culturelle ressentis ${ }^{7}$. Ces particularités sont évidentes dans plusieurs aspects de la tradition orale et, en particulier, dans certaines chansons de composition locale.

Mais malgré l'intérêt que présentent ces particularités, la région du Sud-Ouest avait fait l'objet de très peu de recherches folkloriques avant que j'entreprenne mes enquêtes en 1988. Au

6. Voir surtout Marcel Bénéteau, «Chansons traditionnelles et identité culturelle chez les francophones du Détroit », Ethnologies, vol. 26, n 2, 2004, p. 201-220; et «Variantes phonétiques, morphologiques et lexicales dans le français des deux groupes colonisateurs dans la région du Détroit », Louis Mercier (dir.), avec la collaboration de Hélène Cajolet-Laganière, Français du Canada - Français de France. Actes du sixième Colloque international tenu à Orford (Québec), du 26 au 29 septembre 2000, Tubingen, Max Niemeyer Verlag, 2004, p. 199-212.

7. Voir Marcel Bénéteau, «Singularités et survivances dans le répertoire de chansons traditionnelles françaises du Détroit », Jean-Pierre Pichette (dir.), La Résistance des marges. Exploration, transfert et revitalisation des traditions populaires de francophones d'Europe et d'Amérique, dans Port Acadie, printemps 2008, automne 2008 et printemps 2009, p. 391-405. 
cours des quinze années qui ont suivi, j'ai répertorié plus de 1700 versions de quelque 645 chansons traditionnelles sur l'ancien territoire du Détroit. Ce corpus a été recueilli auprès d'environ 65 informateurs natifs de la région pour la plupart durant les premières décennies du $\mathrm{XX}^{\mathrm{e}}$ siècle. On peut ajouter à ces sources sonores des enregistrements que plusieurs personnes avaient captés de leurs parents, décédés avant que je ne commence mes enquêtes. Une autre source importante du corpus est celle des cahiers manuscrits que plusieurs familles avaient en leur possession : ces cahiers s'étalent sur une période qui va de la fin du XIX ${ }^{e}$ siècle jusqu'aux années 1950. Enfin, quelques sources imprimées - entre autres un journal hebdomadaire, Le Progrès, qui parut à Windsor de 1881 à 1912 - me fournissent bon nombre de chansons additionnelles. À partir de ces diverses sources, j'ai réussi à documenter une bonne partie du répertoire traditionnel de cette population historique.

On ne peut prétendre que ce répertoire est toujours bien connu dans la région : il fait incontestablement partie d'une culture de mémoire, plus que d'un patrimoine vivant. La plupart de mes informateurs sont maintenant décédés et très peu d'entre eux ont transmis leurs répertoires aux générations subséquentes. Depuis le milieu du siècle dernier, l'expansion urbaine, les pressions démographiques et le développement industriel ont complètement bouleversé le contexte dans lequel les chansons se transmettaient; en conséquence, cette génération a été la dernière à connaître ce riche patrimoine. Mon but était en quelque sorte de récupérer un répertoire oublié par les grands folkloristes du Canada français et de combler ainsi une lacune dans les connaissances scientifiques concernant la diffusion du répertoire traditionnel en Amérique française.

Il serait impossible ici de faire une analyse approfondie du corpus. La comparaison des répertoires des deux zones de peuplement entre eux et avec les répertoires recueillis ailleurs en Amérique française relève des particularités relatives aux contextes historiques des deux périodes de peuplement ainsi que 
d'autres phénomènes intéressants ${ }^{8}$. Le présent article propose de laisser l'aspect comparatif de côté pour miser sur une analyse de la forme et de la fonction de la chanson de composition locale.

\section{La place de la chanson locale dans le répertoire}

Le classement du corpus selon les catégories du Catalogue Laforte ${ }^{9}$ nous aide à cerner la place qu'occupe la chanson locale dans l'ensemble du répertoire traditionnel du Sud-Ouest. Le système de classification de Conrad Laforte est basé sur la forme poétique et autres critères formels des chansons traditionnelles et est utilisé par la plupart des chercheurs dans le domaine. Il place les chansons locales dans la catégorie VI, les chansons sur les timbres, c'est-à-dire les chansons composées sur des airs connus. Les chiffres suivants représentent le nombre de chansons types dans chaque catégorie, recueillies dans les deux zones de peuplement, toutes sources confondues :

\begin{tabular}{|c|c|c|}
\hline I- & Chansons en laisse : & 88 \\
\hline II - & Chansons strophiques: & 359 \\
\hline III - & Chansons en forme de dialogue : & 44 \\
\hline IV - & Chansons énumératives : & 63 \\
\hline $\mathrm{v}-$ & Chansons brèves : & 40 \\
\hline & Chansons sur les timbres: & 51 \\
\hline
\end{tabular}

On note immédiatement une proportion relativement faible de chansons de la première et de la quatrième catégorie (chansons en laisse et chansons énumératives), qui ensemble constituent ce qui est communément appelé « chansons à répondre » (23 pour cent). Les proportions sont très différentes pour les répertoires des autres régions de l'Ontario français et de la vallée laurentienne, où ce genre de chanson représente une part beaucoup plus importante du répertoire (presque 60 pour cent, selon Jean-Pierre Pichette) ${ }^{10}$.

8. Voir, entre autres, Marcel Bénéteau, «Singularités et survivances... »; et « Le Cahier chansonnier comme document ethnologique : considérations sur le cahier de Félix Drouillard (vers 1897-1903), Rabaska n 1, 2003, p. 59-80.

9. Conrad Laforte, Catalogue de la chanson folklorique française, Québec, les Presses de l'Université Laval, «Archives de folklore », vol. 1-6, 1977-1987.

10. Voir Jean-Pierre Pichette, « Coup d'œil sur le répertoire traditionnel d'un chanteur franco-ontarien ", Cahiers Charlevoix n ${ }^{\circ} 2$, Sudbury, Société 
En revanche, on remarque dans le Sud-Ouest une prépondérance de chansons narratives, classées surtout dans les catégories de chansons strophiques et les chansons en forme de dialogue (62 pour cent). Les chansons de composition locale se classent pour la plupart parmi les chansons sur les timbres (mais aussi, dans certains cas, parmi les chansons brèves) ; quoiqu'elles constituent une proportion minime du répertoire, elles racontent aussi des histoires et peuvent s'ajouter au nombre de chansons narratives. On peut donc dire que, bien que peu nombreuses, les chansons de composition locale se conforment parfaitement à la préférence de la population pour les chansons narratives dans le répertoire du Sud-Ouest.

\section{Chansons sur les timbres}

Comme cela a été indiqué, le sixième volume du Catalogue Laforte comporte des " chansons sur les timbres », c'est-à-dire des chansons composées sur des airs connus. Cette catégorie suscite parfois de la controverse parmi les experts de la chanson de tradition orale. Le Répertoire de Patrice Coirault ${ }^{11}$, par exemple, n'accepte pas les compositions parfois modernes et d'origine connue qui trouvent leur place dans la sixième catégorie de Laforte. Plusieurs de ces pièces d'ailleurs vont à l'encontre des critères imposés par Laforte lui-même pour la définition d'une chanson traditionnelle : l'auteur, ainsi que le temps et le lieu de la composition sont parfois connus, et la transmission se fait souvent autrement que par voie orale. Certaines chansons classées dans le Catalogue sont nettement de facture lettrée et professionnelle, en particulier celles que Laforte place sous la rubrique $\mathrm{A}$ (Parodies) et $\mathrm{B}$ (Chansons historiques). Mais on ne peut nier le processus folklorique qui encadre la création des chansons

Charlevoix et Prise de Parole, 1997, p. 177-179 pour les caractéristiques du « répertoire laurentien ».

11. Patrice Coirault, Répertoire des chansons françaises de tradition orale, ouvrage révisé et complété par Georges Delarue, Yvette Fédoroff et Simone Wallon, Paris, Bibliothèque nationale de la France, tomes 1-3, 1996-2006. Cet ouvrage développe le seul autre système de classification largement utilisé par les chercheurs, particulièrement en France. 
qu'il place sous la rubrique C (Chansons locales) : l'adhérence à la forme traditionnelle, le calque d'un air traditionnel, la création par un individu non professionnel ou même par une collectivité, la transmission en dehors des réseaux institutionnels, l'intérêt pour des événements qui ont marqué la vie quotidienne de la communauté et, enfin, l'imposition d'une vision du monde qui reflète les valeurs de la communauté. Les chansons locales sont en quelque sorte une interaction avec la tradition et une réinterprétation de celle-ci : il faut connaître la forme, les thèmes, la fonction des différentes sortes de chansons avant de pouvoir en faire une ; il faut maîtriser l'ethnotexte de la région avant de pouvoir participer à ce « discours qu'une communauté tient sur elle-même, son passé, son présent ».

Les chansons de composition locale ne constituent pas une grande proportion du répertoire que j'ai recueilli dans la région du Détroit : une vingtaine de chansons seulement, et ce sur un total de 645 chansons types. D'ailleurs, bien que la majorité des chansons types du répertoire aient été recueillies en versions multiples, les chansons locales sont, à quelques exceptions près, des versions uniques. Il s'agit donc, numériquement, d'une partie presque insignifiante du répertoire. La situation semble assez différente dans d'autres régions francophones. Le cas de l'Acadie est bien connu : en 1937, Marius Barbeau avouait que « le répertoire acadien, beaucoup plus que le laurentien, comprend des pièces de composition locale - peut-être plus de vingt pour cent ${ }^{12}$ ». Georges Arsenault cite ce passage à l'appui de sa propre étude sur les Complaintes acadiennes de l'île-du-Prince-Édouard ${ }^{13}$, étude qui montre la grande vitalité de la tradition dans cette région. Le Catalogue Laforte inclut une cinquantaine de chansons de composition locales provenant des trois provinces maritimes. Le répertoire « laurentien » lui-même n'est pas démuni de chansons locales : le Catalogue en relève 107, qui proviennent de toutes

12. Marius Barbeau, Romancero du Canada, op. cit., p. 184.

13. Georges Arsenault, Complaintes acadiennes de l'Île-du-Prince-Édouard, Montréal, Les Éditions Leméac, 1980. 
les régions du Québec ; si on considère que les 85 chansons politiques enregistrées sont aussi pour la plupart des compositions locales, le total s'approche de 200 pour cette province. Le chiffre est sans doute plus élevé encore, étant donné le manque d'intérêt des chercheurs et le fait que, pour des raisons que nous allons voir, les informateurs sont parfois réticents à les chanter. Le Catalogue ne rapporte aucune chanson locale de l'Ontario ; la collection Germain Lemieux, récemment cataloguée au complet, ne comprend qu'une seule chanson - La Grève des marchands de Chelmsford - pour laquelle on peut confirmer une origine ontarienne $^{14}$. Mais les archives du département de folklore et ethnologie de l'Université de Sudbury, qui comprennent plus de 2000 collections recueillies par les étudiants du département depuis 1981, contiennent une centaine de chansons types qu'on pourrait qualifier de compositions locales, couvrant des sujets aussi variés que des anniversaires et des réunions de familles, des aventures de chasse, des déboires remarquables et des incidents au travail. En comparaison, les chansons locales - particulièrement celles de composition récente - semblent être d'une rareté déconcertante dans le sud-ouest de la province.

Doit-on conclure alors que les gens du Détroit ne faisaient pas des chansons comme les gens d'ailleurs ? Pas nécessairement. Nous pouvons proposer quelques raisons pour cette pénurie dans le répertoire tel que reconstitué à la fin du $\mathrm{xx}^{\mathrm{e}}$ siècle. D'abord, ce genre de chanson a tendance, de par sa nature, à être éphémère. Les chansons portent, pour la plupart, sur des événements ponctuels plutôt que sur des archétypes et des thèmes universels. Étant basées sur des événements locaux, elles « voyagent» moins bien que d'autres chansons. Par conséquent, leur aire géographique est limitée, il y a moins de versions, et leurs chances d'être recueillies

14. Coll. Germain Lemieux $n^{\circ} 529$, chantée par Aldéric Perreault, Sudbury, 1964 ; Centre franco-ontarien de folklore. Le classement de la collection Germain Lemieux - dont seulement le quart figurait au Catalogue Laforte - a été récemment effectué, en collaboration avec Lucie Beaupré et des étudiants du département de folklore et d'ethnologie de l'Université de Sudbury. Ce travail, avec le classement de la collection des Archives de folklore de l'Université et celui de ma propre collection, met à la disposition des chercheurs un fonds de plus de 15000 chansons traditionnelles. 
par un chercheur sont donc moins élevées. Également, puisqu'il s'agit de chansons axées sur des événements et des personnages connus dans la communauté, les gens éprouvent souvent un fort sentiment que les chansons leur appartiennent et ils manifestent une certaine hésitation à les partager avec un étranger. Cette réticence s'explique d'autant plus que certaines chansons étaient composées « sur »-c'est-à dire, au sujet de - des personnes connues dans la communauté ; en général, on ne composait pas de telles chansons pour louer les qualités de quelqu'un. Un de mes informateurs, Richard Bastien, me confia que sa mère «faisait des chansons sur les voisins ». Bien que les voisins en question fussent décédés depuis longtemps, monsieur Bastien jugeait inapproprié de répéter de telles pièces à l'extérieur de la famille. La chanson intitulée Le Déménagement de l'école Robillard, dont je présenterai une version manuscrite dans les pages qui suivent, était toujours connue à Belle-Rivière, mais aucun informateur n'a voulu me la chanter, par crainte d'offenser les descendants de familles impliquées dans des événements qui eurent lieu en 1896.

Je crois aussi que l'état du patrimoine oral dans une région est un facteur préalable à la pratique de " faire des chansons ». Comme on le verra, la facture de chansons exige une bonne connaissance de la forme traditionnelle, du langage textuel et musical des chansons, des thèmes traditionnels et de la façon dont ils sont traités. Le contexte social, les occasions de « tenir un discours sur soi-même ", repose sur des liens étroits entre les membres d'une communauté qui partagent une langue, une culture et des valeurs communes. Le fait que le sud-ouest ontarien est une des régions les plus urbanisées au pays, dans laquelle les francophones sont extrêmement minoritaires face à une majorité non seulement anglophone mais aussi états-unienne, pose plusieurs obstacles à ce genre d'activité. Les institutions scolaires et médiatiques deviennent les principaux transmetteurs de culture, et elles accordent peu de place aux particularités régionales. Dans un tel contexte, il semble évident que les chansons locales sont 
les premières oubliées. D'après mes enquêtes, les gens ne « font » plus de chansons dans la région du Détroit. Ce n'est que le hasard qui nous a conservé un échantillon de ce volet de la tradition.

\section{II - FAÇONS DE FAIRE DES CHANSONS}

\section{Appropriation}

« Faire » une chanson peut signifier bien des choses. On peut proposer une gamme de modes de «fabrication » qui va de l'appropriation ou de la modification de chansons existantes à la véritable création de chansons nouvelles. Les trois démarches répondent à un besoin de s'approprier une tradition reçue et de l'intégrer à son discours identitaire. Au tout début, on peut parler de « fausses attributions », c'est-à-dire des chansons que les gens croient être de composition locale, parfois même attribuées à un individu quelconque. Par exemple, une informatrice de SaintJoachim, à l'est de Windsor, chantait plusieurs pastourelles, mettant en vedette des bergers et des bergères dans des scénarios les plus classiques, tout en affirmant qu'elles avaient été composées par son père au cours de ses longues journées à labourer les champs. Elle ignorait évidemment que les mêmes chansons avaient été recueillies par des chercheurs des deux côtés de l'Atlantique : pour elle, les pièces faisaient tellement partie de la personnalité de son père et des souvenirs qu'elle conservait de lui qu'elles lui appartenaient purement et simplement.

Il ne faudrait pas pour autant prendre une attitude de supériorité devant la naïveté ou la fierté mal placée de certains informateurs. Même les experts se sont parfois laissé prendre au piège. Par exemple, une chanson recueillie par Adélard Lambert, que Conrad Laforte classe parmi les chansons strophiques sous le titre Marie Frisine (II. O-90), semble avoir induit en erreur les plus grands chercheurs : Marius Barbeau a recueilli deux versions de la même chanson à l'île d'Orléans en 1925 et il affirme dans ses notes qu'elle avait été composée à Saint-Tite-des-Caps au sujet d'une certaine « Marie à Job » qui parcourait la région comme colporteuse. Également, Carmen Roy en a recueilli une version 
en Nouvelle-Écosse trente-cinq ans plus tard, notant que le sujet de la chanson serait « Marie Froisine » qui pratiquait autrefois le même métier dans le village de son informatrice ${ }^{15}$. J'ai pourtant repéré la même chanson près de Monroe, au sud de Détroit, dans l'ancienne communauté de Rivière-aux-Raisins (voir la pièce en annexe). L'axe Charlevoix-Acadie-Détroit indique une distribution beaucoup trop large pour une chanson de composition locale (ou pour le champ d'activité d'une colporteuse avec « un œil fait comme un piton » et « les jambes comme une alouette») ; on peut alors postuler que la chanson existait en France avant d'être diffusée à travers l'est du Canada et des États-Unis, même si aucune version ne semble avoir survécu dans la mère patrie.

Il en est de même pour Les Prisonniers politiques (VI. B-55), que Laforte classe parmi les chansons historiques dans le volume du Catalogue consacré aux chansons sur les timbres. Encore ici, on a affaire à une version unique, mais cette fois la généalogie de la chanson semble impeccable : recueillie par É.-Z. Massicotte en 1922, avec l'information suivante notée de son informateur : «Vers l'année 1775, beaucoup de Canadiens furent emprisonnés pour avoir trop parlé contre les Anglais. Un charpentier du Cap St-Ignace, du nom de Clément Gosselin, avait été au-delà d'une année en prison, pour cette cause. Pendant sa captivité, il avait composé la chanson que voici. ${ }^{16}{ }^{\prime}$ Justement le genre de chanson que n'importe quelle communauté aurait été fière de produire, mettant en vedette la valeur et le patriotisme de ses membres. Mais la chose n'est pas si simple : j'ai enregistré la même chanson chez madame Stella Meloche à Rivière-aux-Canards en 1992. Cette nouvelle version, plus complète, ne fait aucune mention d'Anglais ni de politique ; le crime des prisonniers n'est pas spécifié. À moins d'un lien inconnu entre Cap-Saint-Ignace et la rivière Détroit, il est difficile encore une fois d'envisager une telle diffusion pour une chanson locale ; les témoignages

15. Bien que Laforte n'ait répertorié que la version de Lambert, les versions de Barbeau et de Roy sont déposées dans les archives du Musée des civilisations à Gatineau : voir coll. Marius Barbeau, mss 1344 et 1347 et coll. Carmen Roy 33 - (281).

16. Musée national, coll. É.-Z. Massicotte, ms no 915, 1922. 
suggèrent plutôt qu'une chanson française aurait été à l'origine des versions canadiennes. Avec la publication du Répertoire Coirault, j'ai pu confirmer mon hypothèse : Coirault répertorie une chanson recueillie dans le massif central par Victor Smith en 1871, Prisonniers pour des crimes qu'ils n'ont pas faits (6224), qui est sans aucun doute l'antécédent des versions canadiennes (voir les numéros 2, 3 et 4 en annexe).

Ces deux exemples semblent quand même indiquer un besoin des membres d'une communauté de valoriser et de commémorer par la chanson des expériences de leur vécu. Peut-on supposer que Clément Gosselin chantait souvent la chanson, à tel point que les gens de Cap-Saint-Ignace vinrent à l'identifier comme « sa chanson »? Aurait-il lui-même encouragé cette interprétation, ou encore aurait-il modifié la chanson suffisamment pour justifier une appropriation qui reflétait une expérience qui l'avait profondément marqué ? Et que dire de Marie Frisine/Froisine/à Job ? Pourquoi les gens de diverses localités auraient-ils senti le besoin d'associer cette chanson comique à certaines femmes - sans doute marginales ayant vécu dans leurs propres communautés ? Ces deux cas, et bien d'autres semblables, suggèrent que les membres d'une communauté ont éprouvé le besoin de personnaliser un patrimoine reçu en l'associant à leur propre discours identitaire.

\section{Transformation}

De la simple appropriation, on peut passer à la transformation de chansons connues. La transformation peut être minime : par exemple, on ne fait que changer le nom de la localité ou des personnages pour refléter la réalité régionale. Plusieurs de mes informateurs croyaient qu'une des chansons les plus populaires dans le répertoire du Sud-Ouest - Les Filles de Sandwich - était une composition locale ; en fait on m'a suggéré presque autant d'auteurs que de versions ${ }^{17}$. En réalité, il s'agit d'une version de la chanson Les Filles demandent les garçons (II. O-21), qui est

17. Sandwich étant le plus ancien quartier de Windsor, site de l'ancienne paroisse canadienne-française de l'Assomption. 
largement diffusée autant en France qu'en Amérique du Nord, d'après le Catalogue Laforte, avec la particularité que le nom de la localité change à chaque endroit où la chanson est chantée : Les Filles de Belle-Rivière, Les Filles de Prairie-du Rocher, Les Filles de l'Anse du Saint-Balfour, les Filles de Chalençon, etc. Le reste de la chanson varie très peu de version en version (voir une des versions du Sud-Ouest au numéro 5 en annexe). Il en est de même pour la chanson Les Tailleurs de pierre ${ }^{18}$, connue dans le Sud-Ouest comme Les Gens de Sainte-Claire ${ }^{19}$, où « Les grands comme les p'tits, i' boivent toutes du whisky » (voir numéro 6 en annexe). Chanson fort appréciée dans les soirées, le chanteur s'assurait toujours de nommer les personnes présentes parmi « les plus grands débauchés ». Le scénario de la chanson était particulièrement approprié au temps et au lieu de sa plus grande popularité : à la Petite-Côte sur la rivière Détroit pendant la Prohibition des années 1920, lorsque la plupart des habitants étaient engagés activement dans la contrebande de l'alcool aux États-Unis. De plus, le vers « Si on fait une bonne semaine, on vous paiera samedi » était également très adaptable au contexte local, puisque la plupart des familles vivaient principalement de la culture maraîchère, et le samedi était la journée du marché lorsqu'on se rendait à Windsor et à Détroit vendre les produits de la terre. On ne peut donc pas s'étonner que les gens aient présumé une origine locale pour la chanson ; dans d'autres régions du Canada français, la chanson Les Tailleurs de pierre ou Les Gens du chemin de fer était aussi adaptée à un contexte socio-économique local ${ }^{20}$.

18. II. O-106 dans Laforte. Il n'est pas clair pourquoi Laforte l'aurait classée parmi les chansons strophiques et non parmi les chansons sur les timbres, puisque la majorité des versions canadiennes se chantent sur l'air de Sweet Betsy of Pike.

19. Ancien village au bord du lac Sainte-Claire. Le nom Loiselleville qui figure aussi dans la chanson a été le nom donné au village de Rivière-aux-Canards entre 1910 et 1928, en l'honneur de l'abbé Albert Loiselle qui fut curé à cette époque. Le village reprit son nom original après le départ de l'abbé Loiselle.

20. Un cas intéressant de chanson s'adaptant à divers milieux d'occupation a été analysé à fond dans mon article « De Dunkerque à Ford-City, en passant par ces grands bois », "M'Amie, faites-moi un bouquet... ». Mélanges posthumes autour de l'œuvre de Conrad Laforte, sous la direction de Jean-Nicolas De Surmont, Québec, 
La transformation peut aller plus loin que le simple changement de noms. Certains chanteurs sont reconnus pour leur habilité à ajouter leurs propres vers ou strophes à une chanson connue. La chanson originale demeure intacte; pour la plupart, les nouveaux couplets sont ajoutés à la fin de la chanson - parfois de façon spontanée, parfois de façon plus travaillée. Stella Meloche, à la Petite-Côte, qui avait un répertoire de plus de 150 chansons traditionnelles, était quand même très fière d'une chanson qu'elle avait « faite » elle-même. Elle avait, en réalité, composé de nouveaux couplets pour une chanson qu'elle avait entendue à la télévision : Il mouillera plus, pas en toute (VI A-2 au Catalogue Laforte). On peut lire la transcription de sa version pour mieux comprendre sa conception de «faire une chanson » :

Ah, i'mouill'ra pas pantout' pantoute,

I mouill'ra pas pantoute.

La compagnée des parapluies

Va vite aller banqu'rout'.

Quand c'que les p'tites fill's sont p'tites, $I(l s)$ portent des rob's courtes.

Mais quand c'que les p'tit's fill's sont grandes, I(ls) en port'nt pas pantoute.

Quand c'que le manger est sur la table,

Les plus galaff's mangent toute.

Mais quand c'qu'on vient pour se sarvir,

I(l) reste rien en toute.

Quand c'que ma femme $a$ [elle] fait des tartes,

On s'assomme avec les croûtes.

Mais si $a$ [elle] peut pas fair' mieux qu'ça,

M'a m'engager un cook.

Si ma femme est en colère,

J'y parl'rai pas pantoute.

M'en irai drett' dans mon coin

Pour bénir mon chagrin ${ }^{21}$.

Les Presses de 1'Université Laval et Éditions Charlevoix, « Archives de folklore », 2011, p. 151-174.

21. I' mouillera pas pantoute, coll. Marcel Bénéteau 603.28a ; chantée par Stella Meloche le 2 juin 1993, Petite-Côte, Rivière-aux-Canards ; née à l'Île-aux- 
Selon madame Meloche, les deux premiers couplets et le refrain sont ceux qu'elle a appris « sur le television. » La source était une chanson folklorique, mais de transmission tout à fait moderne. Déjà, cette appropriation de strophes préexistantes avait subi un processus de localisation, illustré par l'utilisation du mot galaffre (gourmand), mot du lexique régional qui n'aurait probablement pas figuré dans la version québécoise entendue à la télévision. Elle aurait ensuite composé deux strophes nouvelles. Celles-ci reflètent bien son sens de l'humour et sa façon originale de s'exprimer, mais elles reflètent autre chose encore : une connaissance approfondie de la forme et du langage chansonnier, ajoutant une expression prête-à-porter comme " bénir mon chagrin » (déformation de « bannir mon chagrin »), qui figure dans maintes chansons à boire.

À quel moment la transformation d'une chanson existante aboutit-elle à une nouvelle chanson ? L'exemple suivant, Le Beau Jour de l'An, calqué musicalement et textuellement sur la chanson de noce traditionnelle Le Jour du mariage (II. P-33 au Catalogue Laforte), profite des circonstances semblables des deux événements commémorés, mais se détache suffisamment du contexte original pour présenter, à mon avis, une chanson distincte. Je l'ai recueillie des deux côtés de la rivière Détroit; elle semble inconnue ailleurs dans la francophonie, ce qui me porte à supposer que c'est une variante locale. L'auteur anonyme fait bon usage d'éléments communs aux deux occasions - rassemblement de la famille, repas, consommation d'alcool - pour emprunter sans modification majeure les deux dernières strophes de la chanson; par contre, la deuxième strophe remplace les conseils au mari par l'évocation d'une coutume du jour de l'An, la bénédiction paternell $\mathrm{e}^{22}$. Les changements appropriés sont aussi apportés au refrain et à la première strophe.

Dindes, Rivière Détroit, 1902 ; chanson apprise à la télévision ; deux dernières strophes composées par l'informatrice.

22. On ne peut, cependant, nier la possibilité que cette strophe ait elle-même été empruntée à une autre chanson, jusqu'à présent non identifiée. Cette pratique serait aussi sans doute courante dans la « facture » des chansons de tradition orale. 
Le Jour du mariage ${ }^{23}$

Amis, venez entendre

Cett' chanson qu'j'vas

vous chanter.

C'est pour vous faire comprendre

Que vous devez m'excuser.

Reviens-donc je t'invite,

Profitons du bon temps;

E-rien ne passe si vite

Que l'amour et le temps.

À ton mari ma chère,

Je veux lui recommander,

D'être bon et sincère,

Et de toujours t'aimer.

Le jour du mariage,

C'est un jour très heureux.

Pour un si long voyage,

On est toujours mieux deux.

Nous voilà tous à la table,

Les parents, les amis.

Ce jour-là me ressemble

Au jour qu'on se marie.

Que 1'on m'emporte mon verre

Et ma bouteille de vin,

Que je salue ma bergère

Et toute la compagnie.

\section{Le Beau Jour de l'An ${ }^{24}$}

Mes amis venez entendre

La chanson que je vas vous chanter.

Cela vous fera souvenir

De la nouvelle année.

Oh! reviens donc je t'invite,

Profitons du bon temps.

Rien ne passera si vite

Que le beau jour de l'An.

Un enfant doit se rendre

À son père tous les ans.

Sans aucune rancune,

Tout passe comme le vent.
Nous voilà tous à la table,

Mes parents, mes amis.

Ce jour-là me ressemble,

Le jour qu'on se marie.

Que l'on m'emporte mon verre Et ma bouteille de vin; Que j'en boive à la réserve Avec tous mes amis.
23. Le Jour $d u$ mariage, coll. Marcel Bénéteau 307.01c ; ms. Élisabeth Meloche, Rivière-aux-Canards, 1897.
24. Le Beau Jour de l'An, coll. Marcel Bénéteau 606.02a ; ms. Léa Bondy, Petite-Côte, Rivière-auxCanards, vers 1924. 
Faire un ou deux nouveaux couplets pour une chanson existante n'est pas loin d'en faire un ou deux qui se tiennent comme chanson complète. Le Catalogue Laforte inclut des chansons de ce genre dans le volume consacré aux chansons brèves ainsi que dans le volume des chansons sur les timbres. Le plus souvent, ces chansons sont considérées comme des parodies, présentant un point de vue comique ou satirique sur un membre de la communauté ou sur ses actions. Par exemple, j'ai entendu la chansonnette suivante, composée sur la forme du limerick anglais, d'une informatrice à Pain-Court :

In the shade of the old apple tree,

Le vieux Campbell travaillait pour madame Émery.

Le maudit bâtard

A arrivé une s'maine trop tard,

I(1) était saoul couché dans son lit ${ }^{25}$.

Une autre parodie paraît dans les pages du Progrès, hebdomadaire français de Windsor publié vers la fin du XIX ${ }^{\mathrm{e}}$ siècle. Elle proviendrait d'un correspondant de Rivière-aux-Canards, mais il est difficile de déterminer s'il s'agit d'une publicité pour le magasin de vêtements Rocheleau ou de vers composés pour se moquer d'un jeune homme de la communauté qui la chantait en revenant de la taverne :

C'est le roi Dagobert

Qui a tourné ses culottes à l'envers

Afin de pouvoir aller chez Rocheleau

Acheter du nouveau ${ }^{26}$.

Un dernier exemple fait référence à une élection à Pain-Court, dans le comté de Kent ; selon mon informateur, la " grande gamache » du quatrième vers serait nulle autre que l'ancien premier ministre Brian Mulroney :

25. In the Shade of the Old Apple Tree, coll. Marcel Bénéteau 607.47a ; chantée par madame Claira Peltier-Cleeve, Pain-Court, le 25 août 1995 ; chanson de son père, Wilfred Peltier.

26. Le Bon Roi Dagobert (parodie), coll. Marcel Bénéteau 607.62a ; publiée dans Le Progrès, Windsor, le 22 juillet 1886, p. 1. 
C'est le p'tit club d'la Quatrième, / j'pense bien qu'vous

l'connaissez :

C'est l'vieux Zidas Pelquier [Peltier] puis tous ces assesseurs, S'promèn'nt de porte en porte / chercher ses électeurs :

- Va-t-en donc, grand' gamache, / on l'sait qu't'es t-un menteur ${ }^{27}$ !

Évidemment, faute de versions additionnelles, nous ne pouvons confirmer que ces couplets ne sont pas des bribes isolées de chansons plus longues, bien qu'elles semblent complètes en soi. Elles nous offrent des petits coups d'œil sur la vie de la communauté, mais c'est dans les pièces plus élaborées que nous pouvons réellement aborder certaines particularités locales et régionales. Heureusement, la tradition orale et les sources documentaires nous ont préservé certaines chansons beaucoup mieux développées, pièces qui méritent une analyse plus approfondie.

On peut mentionner une dernière convention avant de parler de chansons nouvelles proprement dites : l'utilisation de ce que Laforte appelle des « moules à chanson », c'est-à-dire des « formules accompagnées d'un refrain qui structure la chanson [...] Ces formules stimulent et orientent l'inspiration du poète populaire ${ }^{28}$. » Un de ces « moules » familiers comprend la formule «Ah oui bien! Vous m'entendez bien! » qui a donné à la tradition orale plusieurs chansons bien connues, comme par exemple Vous m'entendez bien - les pommes de terre (IV. N-11) et Vous m'entendez bien - les curés (IV. Ma-31). L'abbé Charles Laliberté fut curé de la paroisse Saint-Simon et Saint-Jude de Belle-Rivière de 1928 à 1945 ; dans la chanson, la formule « Vous m'entendez bien » a été remplacée par le nom d'une paroissienne non identifiée : « la vieille Desbiens » (le patronyme est toutefois connu à Belle-Rivière) :

27. Le P'tit club de la Quatrième, coll. Marcel Bénéteau 604.18a ; chantée par Raymond Béchard, Pain-Court, le 11 juin 1993.

28. Conrad Laforte, Poétiques de la chanson traditionnelle française, deuxième édition, Québec, les Presses de l’Université Laval, 1993, p. 135. 
Connaissez-vous la vieill' Desbiens ?

Elle est toujours en colère,

Eh ah oui bien

$A^{\prime}$ '[elle] s'fait manger par les punaises,

Ah oui bien, la vieille Desbiens.

Un jour dit à son mari

D'aller charcher le pèr' Laliberté,

Eh ah oui bien!

Pour conjurer tout's ses punaises,

Ah oui bien, la vieille Desbiens.

Quant' le pèr' Laliberté est arrivé,

Les punais's sont tout's cachées.

Avec son grand habersac,

Eh ah oui bien!

Les punaises ont tout's rentré dans les craques,

Ah oui bien, la vieille Desbiens.

Quant' le père' Laliberté fut parti,

Les punaises ont tout's sorti

Cent fois plus mauvaises.

Eh ah oui bien

$\mathrm{I}(1 \mathrm{~s})$ ont attaqué la pérruque

Ah oui bien, d'la vieille Desbiens. ${ }^{29}$

\section{Création}

Nous arrivons maintenant à la création de nouvelles chansons proprement dites. Typiquement, celles-ci sont quand même composées sur des airs connus, ou tout du moins sur des bribes d'airs connus. Une discussion approfondie des transformations musicales implicites dans la facture de nouvelles chansons dépasse malheureusement nos propos ici ; il semble pourtant évident que, comme les transformations textuelles, les mélodies subissent le même processus d'appropriation, de modification et de création. La mélodie de base est souvent choisie en fonction du sujet abordé. Nous prenons à titre d'exemple une chanson

29. Vous m'entendez bien-les poux de madame Desbiens, coll. Marcel Bénéteau 602.16a, chantée par William St-Pierre le 6 juillet 1994 à Tecumseh ; né à BelleRivière, 1900 ; chanson de sa mère, Rose Plante-St-Pierre. 
qui nous permet de mettre en évidence à la fois la technique de composition ainsi que la signification personnelle et sociale de la chanson de composition locale.

La chanson nommée Départ et retour du soldat de Sandwich m'est parvenue par l'entremise de Margaret Soullière, petite-fille du chanteur et présumé auteur de la chanson. Selon la tradition familiale, Félix Soullière l'aurait composée pour les funérailles d'un de ses camarades d'armée tué en France lors de la Première Guerre mondiale. La version a été enregistrée sur microsillon par un membre de la famille et plus tard transférée sur disque audio numérique. La chanson relate peu de faits historiques comme tels, mais décrit les impressions d'un jeune homme partant pour la guerre. Elle est en fait composée de ce que Jean-Michel Guilcher a nommé des éléments de «prêt-à-porter langagiers », phrases et expressions figées qui sont transférables d'une chanson à une autre : " les incipit, les articulations narratives, les formules récurrentes, la phraséologie, la plasticité et la fonction des clichés, la nature du lien entre le narratif et le dramatique [...] etc., etc. ${ }^{30} »$. Les expressions faut quitter le pays/Je reviendrai au pays, adieu donc tous mes bons parents, c'était pour bien longtemps, retire plus en arrière, je reviendrai au pays, dessur le champ, c'est de t'y voir partir (avec emploi de y), le mot bâtiment pour « bateau » ou « navire », le départ pour aller voir la ville de Paris, pour battre les Allemands, l'adieu à sa chère petite toutes ces locutions figurent communément dans les chansons de soldats et de marins et d'exilés ; pour composer une chanson de guerre, il faut évidemment parler ce langage. En même temps, la chanson contient plusieurs prononciations régionales (mouman « maman », yerre « guerre », $f i$ « fils », etc.), établissant un lien important avec l'identité locale des auditeurs de la chanson.

Le timbre choisi est parfaitement approprié à la chanson :

30. Jean-Michel Guilcher, "Les collecteurs du XIX ${ }^{\mathrm{e}}$ siècle ont-ils inventé la chanson folklorique? », Jean-Loïc Le Quellec (dir.), Collecter la mémoire de l'autre, Niort, Geste Éditions, 1991, p. 26. Voir aussi, du même auteur, La Chanson folklorique de langue française. La Notion et son histoire, Paris, L'Atelier de la danse populaire, 1985, en particulier la section «Phraséologie » (p. 111-139). 
celle-ci est calquée plus ou moins sur l'air de La Fille matelot (II. C-9), une des chansons les mieux connues du répertoire du sud-ouest ontarien. Elle s'impose donc naturellement comme mélodie porteuse d'un voyage sur mer. Mais ce n'est pas une copie exacte de la forme musicale de l'originale ; plutôt, ce sont des bribes de mélodie, des phrases musicales tronquées et répétées qui évoquent la chanson modèle et qui placent le voyage du jeune soldat dans le contexte de tous les voyages sur mer pour aller au combat. Il semble que ce serait précisément le but de ce genre de chanson qui, avouons-le, n'est pas un ouvrage littéraire des plus accomplis, mais qui sert à mettre un événement réel et personnel dans le contexte d'un scénario traditionnel bien connu : le départ du soldat. En utilisant un modèle narratif et musical commun, qui parle un langage déjà maîtrisé et propice à l'expression des sentiments, la pièce se sert de la tradition pour donner un sens à une expérience tragique. Elle permet à la communauté de commémorer l'événement et au chanteur d'inscrire son expérience personnelle dans la conscience collective :

Six mois sont finis

On quitt'ra le pays.

Six mois finis,

Faut quitter le pays

Pour aller voir la ville de Paris

J'arrive pour dire adieu,

Adieu tous nos amis.

- Mon cher ami,

Tu vas, tu me le dis,

Tu m'y fais peur,

Tu m'y fais mal au cour,

C'est de t'y voir partir

Pour aller à la yerre.

- Ma chère petite,

Je vous le dis encore,

Je reviendrai ici au pays.

Nous metterons ensemble

Pour le rest' de la vie.
Il y a quelqu' chose de plus coûtant, C'est d'aller dire adieu à ma chère mouman :

- Adieu mon père,

Oh adieu ma chère mère, Adieu donc tous mes bons parents. Je viens vous dire adieu C'était bien pour longtemps.

- Mon divin $f i$ [fils], Mon charmant enfant, Tu t'en vas donc Dans les pays si grands. Je prierai Dieu pour toé Qu'i' te ramène à moé. 
- Mouman, ma mère,

Retire plus en arrière :

Il faut aller

Avec-que les Alliés,

Pour battre les ennemis

Qui veulent prend' not' pays.

De ville en ville,

$\mathrm{Ah}$, nous voilà rendus,

Su' l'bâtiment,

Pour passer l'océan.

Je vous assur', mes chers,

Que j'ai plus d'agrément.

On arrive en France,

C'est là qu'i' nous demandent,

- Vous v'nez ici

Pour défend' vot' pays,

Mais là mes chers amis, Ainsi que vos fusils.
De ville en ville,

Ah nous voilà rendus,

Dessur le champ,

Pour battre les Allemands.

Ça va donner,

On va les désarmer.

Oh grâce à Dieu,

Le plus haut des cieux,

C'est le grand roi,

C'est lui qui fait la paix,

Le plus grand des rois,

C'est lui qui fait la paix.

[.......................?]

Mes bons amis,

La yerre est finie.

Mon nom c'est Médéric,

J'm'en r'tourne à Sandwich.

On doit ajouter quelques mots sur le « Médéric » qui s’identifie comme narrateur de la chanson, bien qu'on sache que le chanteur est Félix Soullière. Si monsieur Soullière en est bel et bien l'auteur, pourquoi aurait-il choisi le nom de Médéric dans la «signature » de sa chanson? Parce que le nom aurait la même assonance que « Sandwich »? Pour se donner une certaine distance de son sujet, renforçant en quelque sorte la notion que la chanson est plus qu'une expression personnelle, qu'elle appartient véritablement à la tradition ? On ne saurait répondre à ces questions. Germain Lemieux, qui a abordé le sujet des « chansons signées » dans la tradition orale, est de l'avis que ces signatures n'ont aucune valeur historique $^{31}$. Selon lui, il s'agit simplement de formules utilisées pour clore une chanson : "Alors que la plupart des chansons de table se terminent par une invitation à "prendre un coup", que les chansons de retour du soldat se terminent souvent par une décision de retourner au régiment, tout un autre groupe de chansons réserve

31. Germain Lemieux, Chanteurs franco-ontariens et leurs chansons, Sudbury, La Société historique du Nouvel-Ontario, « Documents historiques » $\mathrm{n}^{\text {os }} 44-45,1963-$ 1964, p. 71. 
un couplet pour nommer l'auteur de la chanson ${ }^{32}$. » La formule commence souvent - comme on verra dans certaines autres chansons du Sud-Ouest - avec « Qui a composé la chanson... », mais les paroles qui suivent ne constituent pas nécessairement une preuve de l'identité de l'auteur.

\section{III - La Communauté du Détroit vUe PaR les Chansons}

Ayant proposé une gamme de factures de chansons passant par l'appropriation, la transformation et la création, processus qui sert à rattacher la communauté actuelle à un patrimoine culturel beaucoup plus large, je vais maintenant présenter un répertoire de chansons qui mérite une attention spéciale pour ce qu'il peut apporter à nos connaissances sur certains aspects historiques et culturels de la communauté francophone du Sud-Ouest. Je vais d'abord considérer des chansons d'intérêt historique, y compris deux chansons d'élection. Dans un deuxième temps, je me pencherai sur des chansons qui portent sur les mœurs et coutumes et les conditions sociales de la communauté du Détroit. Les textes complets de toutes ces chansons se trouvent en annexe.

\section{Chansons historiques et politiques}

\section{Les Mascoutens}

La chanson Les Mascoutens ( $n^{\circ} 7$ en annexe) m'a été chantée en 1992 par Stella Meloche à la Petite-Côte, au sud de Windsor. Née en 1902 à l'Île-aux-Dindes dans la rivière Détroit, où son père Henri Meloche était gardien de chasse, madame Meloche a été une de mes informatrices privilégiées, chantant au cours d'une douzaine de rencontres de 1992 à 1994 plus de 150 chansons tirées de sa mémoire prodigieuse ; elle fut aussi une source importante de renseignements sur plusieurs aspects de la vie traditionnelle du Détroit (entre autres dans les domaines de la pêche et la chasse, la médecine populaire et le lexique régional). Elle avait appris la chanson de son père et de son grand-père, Étienne Meloche, ce dernier étant né en 1828. La chanson, qui date sans doute du

32. Ibid., p. 69. 
$\mathrm{XVIII}^{\mathrm{e}}$ siècle, semble inconnue ailleurs dans la francophonie ; la référence aux Mascoutens, alliés des Renards et, comme eux, originaires de l'ouest du lac Michigan, suggère fortement que la chanson aurait été composée dans la région. Les Mascoutens faisaient partie d'un groupe d'Amérindiens qui assiégèrent le fort Pontchartrain du Détroit en 1712 et qui furent par la suite massacrés par les Français et leurs alliés. Les survivants de la bataille s'acharnèrent pendant toute une génération à harceler les expéditions commerciales des Français le long des cours d'eau menant au Détroit. Il est fort possible que la chanson ait compris autrefois des strophes additionnelles, mais la découverte de nouvelles versions s'avérant fort douteuse, nous devons tenter une interprétation basée sur la performance de madame Meloche.

Le scénario semble décrire un affrontement entre les Français et un groupe de Mascoutens qui emploient une ruse pour déjouer leurs ennemis : un « sauvage » donne l'alerte aux Français, qui se mobilisent contre une attaque imminente des Iroquois; pendant que les Français sont ainsi préoccupés, les Mascoutens s'emparent de leur butin. Selon madame Meloche, certains mots dans la chanson seraient des vocables amérindiens. Quoique sans doute déformés par la transmission orale, au moins l'un d'eux semble authentique : patago, qui dans le contexte de la chanson signifierait « méchant, malin »; selon Baraga ${ }^{33}$, le préfixe bataimplique la connotation de péché, mal, ou dommage en langage ojibwé ; dans les langues algonquines, la racine pata- signifie mal, à tort ; patatowin signifie péché ${ }^{34}$. Le mot patago renforce donc le sens de «malin » dans la chanson.

La chanson est intéressante en ce qu'elle témoigne de la place du Détroit aux XVIII et XIX ${ }^{\mathrm{e}}$ siècles comme chef-lieu et point de départ pour les territoires français du centre-ouest états-unien et son appartenance à l'aire culturelle mitoyenne décrite par Richard

33. FredericBaraga,ADictionary of the Otchipwe Language explainedinEnglish, Montréal, Beauchemin et Valois, 1881, p. 70b.

34. Jean-André Cuoq, Lexique de la langue algonquine, Montréal, J. Chapleau et fils, 1886, p. 330a. 
White dans The Middle Ground ${ }^{35}$. Elle nous peint un portait intéressant de la vie à cette époque d'alliances entre les Français et les Amérindiens.

\section{Maudit Lincoln}

Cette chanson ( $\mathrm{n}^{\circ} 8$ en annexe) se rapporte à la Guerre civile aux États-Unis et témoigne elle aussi du caractère unique de la communauté du Détroit à la frontière de la francophonie canadienne. La pièce nous fournit également un exemple frappant de l'éclairage que la tradition orale peut apporter à notre compréhension de certains aspects d'une communauté. Le message n'est pas toujours positif : peu de sources historiques ont commenté le racisme dans la société traditionnelle canadiennefrançaise.

La chanson nous provient d'un enregistrement fait par Ted Drouillard de ses parents, Moïse et Emma Drouillard, qui habitaient à Riverside, à l'est de Windsor, en 1970. Ce quartier résidentiel, fusionné à la municipalité de Windsor en 1965, fut peuplé auX XVIII et $\mathrm{XIX}^{\mathrm{e}}$ siècles par des colons de langue française ; les Drouillard étaient une des familles pionnières de la région. Moïse Drouillard (né en 1883) épousa Emma Peters (née en 1890) de Détroit ; le patronyme de celle-ci était à l'origine Pitre, et de provenance acadienne. Une forte présence francophone persista à Détroit jusqu'aux premières décennies $\mathrm{du} \mathrm{Xx}^{\mathrm{e}}$ siècle et cette alliance témoigne des liens étroits entre les populations des deux rives. Notons que la composition locale de la chanson ne peut être absolument confirmée, puisque aucune référence interne ne la relie à la région. Mais la tradition orale anglo-américaine foisonne de chansons anti-Lincoln et une forte présence francophone à Détroit à cette époque en fait un endroit logique pour l'origine d'une chanson française à ce sujet ; au moins, on peut supposer qu'il s'agit d'une traduction ou d'une adaptation locale d'une chanson anglaise. En plus d'employer plusieurs expressions et

35. Richard White, The Middle Ground. Indians, Empires and Republics in the Great Lakes Region, 1650-1815, Cambridge, Cambridge University Press, 1991. 
prononciations typiques du français régional, la chanson s'accorde très bien avec la situation politique de Détroit à l'époque.

Les relations entre Noirs et Blancs étaient très tendues à Détroit pendant la Guerre civile. On imagine souvent que le Nord était unanime dans son opposition à l'esclavage, mais la réalité s'avère plus compliquée. Le Detroit Free Press, journal partisan du parti démocrate, prend une position fortement antirépublicaine et carrément anti-noire ${ }^{36}$. Détroit, comme plusieurs autres villes du nord des États-Unis, fut le site d'une violente émeute contre les Noirs en 1863 ; il y eut à la même époque plusieurs autres manifestations anti-conscription menant même au lynchage. Bien que Windsor, comme terminus du chemin de fer clandestin, accueillît des Noirs fuyant l'esclavage du Sud, le racisme existait bel et bien des deux côtés de la rivière et les Canadiens français n'en étaient pas exempts.

Cette chanson rare révèle un aspect « invisible » de la communauté et nous permet de constater des attitudes qui sont loin de celles du « folklore officiel » qu’on préfère célébrer.

\section{Déménagement de l'école Robillard}

Une troisième chanson historique relève d'un dossier plus typiquement franco-ontarien, mais sous un angle tout à fait conforme à la situation linguistique du Sud-Ouest. La chanson provient d'un cahier manuscrit rédigé à Belle-Rivière en 1930 par un jeune adolescent nommé Léo Plante. Monsieur Plante est décédé à l'âge de quinze ans et son cahier a été précieusement gardé par son frère Edgar, âgé de 74 ans lors de notre rencontre en 1994.

Le Déménagement de l'école Robillard ( $\mathrm{n}^{\circ} 8$ en annexe) raconte un incident qui présage les grandes luttes scolaires du $\mathrm{XX}^{\mathrm{e}}$ siècle. Aucune source historique ne semble avoir retenu l'événement, si ce n'est l'album du cent cinquantième anniversaire

36. Voir, par exemple, l'article de Matt Kundinger, « Racial Rhetoric: The Detroit Free Press in the Detroit Race Riot in 1863 », Michigan Journal of History, hiver 2006. http://www.umich.edu/ historyj/pages_folder/articles/Racial_Rhetoric.pdf. 
de la paroisse Saint-Simon et Saint-Jude de Belle-Rivière ${ }^{37}$. Il serait impossible ici de résumer la trame des conflits et crises scolaires en Ontario qui aboutirent au Règlement XVII en 1912 ; plusieurs historiens se sont penchés sur ce dossier compliqué et leurs travaux pourraient sans doute nous éclairer sur le contexte du déménagement de l'école Robillard ${ }^{38}$. Les circonstances semblent être les suivantes, d'après les renseignements tirés de l'album souvenir et le témoignage de mes informateurs. L'école en question, située dans le village de Belle-Rivière à l'est de Windsor, avait été établie par le père Pierre Point entre 1843 et 1856. Elle devint une école publique en 1870, bien que la loi Scott de 1863 autorisât l'existence d'écoles confessionnelles. Les paroissiens semblaient toutefois satisfaits de cet arrangement, puisque l'ensemble des enseignants et des élèves était catholique et que l'instruction avait lieu en français. Mais, en 1896, le curé Edmond Meunier décida d'exercer à nouveau le droit des catholiques d'avoir leurs propres écoles et entreprit des démarches pour transférer l'école au système séparé. Un groupe de paroissiens, craignant que le transfert n'occasionne une augmentation de leurs impôts, se rassembla une nuit et organisa une corvée pour mettre l'école sur des rouleaux et la transporter à quelque quatre kilomètres vers le sud, à l'extérieur des limites de la municipalité. On la déposa sur le terrain de la ferme Robillard, où elle demeura publique et fut connue comme « l'école Robillard » jusqu'à sa fermeture en 1961.

La chanson raconte le déménagement et ses conséquences, événement qui semble avoir profondément divisé la communauté. L'abbé Meunier prêcha contre les rebelles en chaire le jour même de Noël, ce qui incita certains des conspirateurs à quitter l'office ; une des strophes suggère même que le curé leur aurait refusé

37. The Parish Family of St. Simon and St.Jude. 1834-1984. La Famille paroissiale de St. Simon et St.Jude, 1984, p. 130-133.

38. Voir, entre autres,Albert Plante, s.j.,Les Écoles séparées d'Ontario, Montréal, Collection « Relations » $\mathrm{n}^{\circ}$ 3, 1953, 103 p. ; et plus particulièrement Jack Cécillon, «Early Struggles for Bilingual Schools and the French Language in the Windsor Border Region, 1851-1860 », Historical Studies in Education/Revue de l'histoire de l'éducation, printemps/été 2009, p. 66-84. 
l'absolution au confessionnal. Au-delà de l'intérêt historique, la chanson est intéressante à plusieurs égards. L'auteur nomme les participants au complot et nous fait part de plusieurs sobriquets intéressants, comme Bébé Meunier, Crapaud et Garçon Girard ; les couplets nous laissent également entrevoir les relations dynamiques entre les membres de la communauté. Il est intéressant de noter que la chanson tente de valoriser l'action des paroissiens en reliant leur rébellion aux Rébellions de 1837, profitant de la présence de personnes nommées Papineau impliquées dans le déménagement de l'école. La composition semble en fait s'inspirer d'une chanson de la Rébellion, Ah!Ah! Ah! Mais cependant (VI. 8.17.1 dans Laforte) ; la version unique répertoriée par Laforte aurait paru dans La Patrie en 1933, et n'aurait donc pu servir de modèle pour la chanson de Belle-Rivière. Mais É.-Z. Massicotte en avait publié une version partielle dans le Bulletin des recherches historiques en $1921^{39}$, ce qui indique que la chanson circulait depuis quelque temps dans la tradition orale. Comme son modèle, notre chanson est probablement chantée - en prenant certaines libertés prosodiques - sur l'air de Cadet Roussel ; le refrain «Ah ! Ah ! Ah ! Mais cependant/nous en rirons bien longtemps » de la version originale est ici modifié en « Mais cependant/Monsieur Papineau fournira son temps. » Le fait que la pièce originale était une chanson « anti Papineau » ne change pas l'intention de transmettre un peu de l'héroïsme du protagoniste aux participants.

Il est à rappeler que la chanson de l'école est encore connue de nos jours par certains chanteurs, mais que, malgré son consentement initial, l'informateur que j'ai contacté a décidé de garder le silence, de peur d'offenser les descendants des personnes nommées.

\section{Trois chansons politiques}

Trois chansons politiques nous sont également parvenues de

39. É.-Z. Massicotte, « Papineau et la chanson », Bulletin des recherches historiques, vol. 27, janvier 1921, p. 22. Cette version est absente du Catalogue Laforte. 
Belle-Rivière et Tecumseh par l'entremise du journal Le Progrès. La première, parue en 1884, s'intitule Le Pier de Belle-Rivière et raconte un incident de corruption autour de la construction d'un nouveau quai au port de Belle-Rivière. Le « $\mathrm{McCu}$ » de la chanson est sans doute le chef d'une compagnie de construction locale du nom de McHugh ; la Rivière-à-Peck (aujourd'hui Pike Creek) était un village avoisinant Belle-Rivière (voir $\mathrm{n}^{\circ} 10$ en annexe).

Deux autres chansons relatent les résultats locaux de l'élection provinciale de 1902. Le journal Le Progrès, publié par les frères Aurèle et Gaspard Pacaud, avait été établi à Windsor en 1881 dans le but de faire une percée dans le sud-ouest ontarien pour le parti libéral ; rien dans ses plus de trente ans d'existence ne suggéra une abrogation de sa mission hyper partisane - sauf lors de l'élection de 1902, où les frères jugèrent le candidat libéral, William McKee, hostile à la cause canadienne-française. Cette cause plus élevée les incita donc - pour une seule fois - à appuyer le candidat conservateur francophone, Alex Réaume. Deux chansons célèbrent sa victoire et la défaite de McKee. La première, Défaite de McKee à Belle-Rivière, nomme les partisans du parti libéral dans cette communauté ( $\mathrm{n}^{\circ} 11$ en annexe). Le style de composition est tout à fait traditionnel, employant plusieurs formules bien connues comme l'incipit Écoutez je vas vous chanter/Une chanson de vérité, et des formules énumératives comme parlons donc de... et revenant à... La deuxième chanson, signée « un conservateur de Tecumseh » fait appel dans sa dernière strophe au verre et flacon qui est la façon typique de conclure une chanson de circonstance, une façon d'insérer un événement ponctuel et local dans la tradition orale ( $\mathrm{n}^{\circ} 12$ en annexe).

\section{Chansons sur la vie sociale et les conditions civiles}

Certaines chansons du répertoire nous ouvrent une fenêtre sur des événements touchant de plus près à la vie quotidienne d'autrefois. En même temps, elles permettaient aux membres de la communauté de commenter directement les conditions sociales en général et plus spécifiquement le comportement de certains individus. 


\section{La vendeuse d'oufs}

La vendeuse d'œufs provient d'un cahier manuscrit rédigé par Edwidge Monforton à la Petite-Côte en 1908 (nº 13 en annexe). La chanson traite un sujet typique de la vie à la frontière : la contrebande, ou le smuggling. Occupation commune à toute région frontalière, l'activité est encore plus intégrée à la vie quotidienne lorsqu'une frontière est créée après l'établissement d'une population. Le peuplement français était bien établi des deux côtés de la rivière Détroit presque un siècle avant la création de la frontière internationale en 1796 ; cet événement politique n'a pas réussi à diviser les familles habitant les deux rives de la rivière. Encore aujourd'hui, les gens d'un certain âge ne disent pas « les États-Unis » pour désigner le côté « américain » de la rivière, mais simplement « l'autre bord».

La chanson décrit, de façon humoristique, un incident mineur de contrebande ratée, composée sans doute pour commémorer une aventure réelle d'un membre de la communauté. Je n'ai recueilli aucune autre version de cette chanson; on peut supposer que sa vie fut assez éphémère et sa diffusion limitée aux gens connaissant la personne en question. Mais la chanson offre un beau témoignage d'une des réalités de la vie dans cette zone liminaire. Dans un article publié dans Canadian Folklore Canadien, «Smuggling Across the Windsor-Detroit Border : Folk Art, Sexual Difference and Cultural Identity ${ }^{40} "$, Janet Langlois décrit bien le rôle de la contrebande dans la construction identitaire des habitants de Windsor et Détroit. Selon elle, l'activité est une forme d'expression populaire qui met en valeur les tensions identitaires dans l'espace marginal que représente la frontière. Elle souligne plusieurs modalités de la contrebande et relie les méthodes et les objets utilisés au sexe des contrebandiers : denrées alimentaires et vestimentaires cachées sous les vêtements pour les femmes, contrebande d'alcool et tabac en bateau ou en véhicule pour les hommes. Certains contrebandiers entreprenants auraient même

40. Janet Langlois, " Smuggling Across the Windsor-Detroit Border : Folk Art, Sexual Difference and Cultural Identity », Canadian Folklore Canadien, vol. 3, n 1 , 1991, p. 23-33. 
réussi à smuggler des maisons entières et leur contenu d'une rive à l'autre ${ }^{41}$. La tradition orale locale abonde en légendes concernant les « bootleggers », c'est-à-dire les contrebandiers d'alcool à l'époque de la prohibition aux États-Unis - vocation pour laquelle les pêcheurs et chasseurs francophones de la rivière Détroit étaient particulièrement bien préparés.

La smuggleuse de notre chanson se voit clairement comme la victime dans cet incident, comme si elle avait été privée d'un droit fondamental. Cette perception ne va pas contre l'opinion populaire. Le Progrès publie régulièrement à cette époque des articles et des éditoriaux dénonçant le zèle des douaniers, qu'on voit comme dressant des obstacles déraisonnables au commerce naturel entre les deux rives. Par exemple, plusieurs Canadiennes françaises de la Petite-Côte augmentaient les revenus de leurs familles en tressant de la paille pour la manufacture de chapeaux de paille et autres objets vendus dans les grands magasins de Détroit ; les tarifs dus à la frontière pouvaient réduire considérablement leurs profits, ce qui provoquait des solutions originales : dans un article du 2 mars 1885, Le Progrès rapporte un incident dans lequel un douanier de Détroit donne la chasse à une dame Odette de Rivière-aux-Canards, la jugeant « un peu trop grosse »; une fouille éventuelle révélera effectivement qu'elle a« enroulé autour d'elle 423 verges de paille ${ }^{42} »$ !

La contrebande, dans toutes ses manifestations, fait partie du discours identitaire de la population francophone du Détroit. On pourrait même qualifier le smuggling de véritable rite de passage - concret aussi bien que symbolique - pour tout habitant d'une zone frontalière. La Vendeuse d'œufs reflète donc un aspect tout à fait typique de la vie des francophones du Sud-Ouest.

\section{Monsieur Prieur}

Si la chanson précédente met en vedette une personne locale mais anonyme pour faire un commentaire sur une réalité régionale, les

41. Ibid., p. 30.

42. Le Progrès, Windsor, le 2 mars 1885, p. 2. 
deux pièces qui suivent sont des exemples de chansons faites sur des individus particuliers qui se sont mérité la désapprobation de la communauté. La première, Monsieur Prieur (n 14 en annexe), est tirée du cahier manuscrit de Félix Drouillard, rédigé à Rivièreaux-Canards entre 1897 et $1903^{43}$. Les patronymes mentionnés dans la chanson appartiennent tous à des familles locales. Bien que la «signature » et l'insertion de noms connus ne soient pas toujours, comme on l'a vu, des preuves fiables de composition locale, la banalité des événements décrits aussi bien que le curieux mélange de détails à la fois particuliers et opaques dans la relation des bêtises du héros suggèrent fortement que la pièce est destinée à un auditoire privilégié qui a souvent entendu parler des incidents et des personnages en question. L'absence de versions recueillies ailleurs renforce la notion que ce n'est pas le genre de chanson qui aurait bien « voyagé ». Monsieur Prieur attire les railleries de ses voisins en raison de son profond manque de savoir-faire et de savoir-vivre. La chanson suivante, par contre, répond à des transgressions beaucoup plus sérieuses.

\section{Le Vieux Baptissette Bebi}

J'ai recueilli cette chanson auprès d'Ubald (Slim) Ducharme, un informateur natif de Tecumseh à l'est de Windsor ( ${ }^{\circ} 15$ en annexe). Selon lui, il s'agit d'une chanson « vraie » qui a été faite « sur les gens du petit Trécarré ». La chanson décrit un incident de violence familiale qui eut lieu vers le début du $\mathrm{Xx}^{\mathrm{e}}$ siècle. Un dénommé Baptissette Bebi - sobriquet de Baptiste Soullière, d'après monsieur Ducharme - bat sa femme pour avoir organisé une veillée malgré son opposition à ce genre de divertissement. La chanson surprend par sa description de la violence commise contre la femme ; il est évident que la communauté n'approuve pas cette action et les paroles peuvent être vues comme une censure sévère de ce genre de comportement.

Mais la désapprobation de la communauté ne s'arrête pas à la condamnation de la violence conjugale. Comme la chanson

43. Collection Marcel Bénéteau, manuscrit nº 22 . 
l'indique, les voisins avaient participé à une corvée et la tradition exigeait que ce genre d'entraide soit payé par une soirée. La coutume était bien connue au Canada français ; par exemple, Georges Arsenault explique au sujet des frolics (corvées) à l'Îledu-Prince-Édouard : «Pour qu’un frolic soit bien réussi, il devait absolument se terminer par un bon repas - souvent un fricot au poulet ou une râpure substantielle - et, évidemment, par de la musique et de la danse ${ }^{44} \gg$. Jeanne Pomerleau, qui cite Arsenault dans son ouvrage sur les corvées et les quêtes, donne plusieurs exemples tirés de toutes les régions canadiennes ${ }^{45}$. Madeleine Ferland-Doyon commente aussi cette tradition et les sanctions associées dans un article sur les rites de voisinage, soulignant que « le peuple, ignorant les lois établies par le pouvoir civil, sait se rendre justice en châtiant par la ruse ou la moquerie les infractions aux coutumes établies ${ }^{46} »$. Sur le plan social, la transgression de gens qui refusent leur obligation de « voisinage » est tout aussi sérieuse que l'homme qui bat sa femme : ils « Nous ont fait des bêtises/Ça leur sera payé ».

Cette chanson illustre bien une autre fonction de la chanson de composition locale : celle de la sanction sociale, une façon de censurer les gens qui agissaient en dehors des normes acceptées. Selon le témoignage de plusieurs informateurs, ce genre de chanson était relativement commun, mais j'en ai recueilli très peu ; en général, ce ne sont pas des chansons qu'on chantait à des étrangers.

\section{La Chanson du Détroite : Une chanson qui a "voyagé »?}

Je termine ce survol de chansons locales du Sud-Ouest en proposant une composition qui aurait peut-être transcendé les limites normalement imposées à la diffusion géographique de ce

44. Georges Arsenault, Guide historique de la région Évangéline, Île-du-PrinceÉdouard, Moncton, G. Arsenault, 1994, p. 33-34.

45. Jeanne Pomerleau, Corvées et quêtes. Un parcours au Canada français, Montréal, Éditions Hurtubise, 2002 ; voir surtout p. 33-39.

46. Madeleine Doyon, « Rites de voisinage chez trois populations rurales canadiennes(Beauce, Dorchester etCharlevoix)», Coutumes populaires du Canadafrançais, Québec, Presses de l'Université Laval, 1972, p. 143. 
genre de chanson. En 1919, Marius Barbeau enregistra à SaintAntonin, Temiscouata, une pièce qu'il nomma « La Chanson du Détroite » (voir no 16 en annexe). Son informateur, Ovide Soucy, l'avait apprise de son père quarante ans plus tôt. Selon Barbeau, cette petite chanson d'amour déçu serait une « chanson de composition canadienne ; un ancien modèle français a servi de base à cette chanson ${ }^{47} \gg$. Deux des strophes portent l'incipit «C'est dans le bord du Détroite... », employant une ancienne prononciation de « Détroit» (on lit par exemple dans un document du gouverneur Livingston de New York : «The best way to effect this is to build a fort at Wawyatchtenok called by the French De Troett the most beautiful inland place in America... ${ }^{48}$ » cf. les prononciations étrouette, étrette « étroit » et drette « droit», toujours employées couramment dans la région). Il est intéressant que le chanteur n'ait pas changé « le Détroite » pour « le SaintLaurent »; on peut postuler que, pour lui, le nom était une partie intégrale de la chanson.

On peut par contre se demander si « le Détroit » en question est effectivement le Détroit du lac Érié ou s'il s'agit d'un autre passage d'eau. É.-Z. Massicottte a recueilli la même chanson en 1917 auprès de Vincent-Ferrier de Repentigny ; dans cette version, il n'y a aucune référence au Détroit et l'action se déroule « tout autour de ces îlettes » ( ${ }^{\circ} 17$ en annexe). J'ai discuté ailleurs des liens surprenants entre le répertoire du Détroit et celui de monsieur de Repentigny, résultat sans doute de son séjour dans les chantiers forestiers du Michigan ${ }^{49}$; soulignons aussi que le mot îlette pour désigner " petite île » est attesté au Détroit depuis le XVIII ${ }^{\mathrm{e}}$ siècle et figure toujours dans le parler régional ${ }^{50}$. La chanson, qui est

47. Note accompagnant la transcription de «La chanson du Détroite », coll. Marius Barbeau n 1962, juin 1919, Musée National.

48. Cité dans E. B. O'Callaghan, Documents Relating to the Colonial History of the State of New York, 1856-1861, vol. IX, p. 363.

49. Voir Marcel Bénéteau, « Le Cahier chansonnier comme document ethnologique [...]», op. cit., p. 70 .

50. Voir Marcel Bénéteau et Peter Halford, Mots choisis. Trois cents ans de francophonie au Détroit du lac Érié, Ottawa, Les Presses de l'Université d'Ottawa, 2008, p. 286-287 ; voir aussi France Martineau et Marcel Bénéteau, Incursion dans le 
absente du Catalogue Laforte, semble inconnue ailleurs sauf pour deux autres versions que j'ai recueillies dans le Sud-Ouest. La première provient du cahier manuscrit de Félix Drouillard de la fin du XIX $x^{e}$ siècle ; la deuxième, plutôt fragmentaire, m'a été chantée par Stella Meloche. Ces deux versions ne nomment pas « le Détroit» mais situent l'action simplement « au bord de la rivière » et « la belle rivière »; si la chanson est véritablement de composition locale, les chanteurs du Détroit n'ont peut-être pas ressenti le besoin de nommer le cours d'eau en question, puisque tout le monde aurait compris la désignation de la «belle rivière » (voir la version de Félix Drouillard, ${ }^{\circ} 18$ en annexe).

Compte tenu de ces témoignages linguistiques et historiques, on ne peut exclure la possibilité que la chanson ait été composée au Détroit ; il n'est pas déraisonnable non plus de supposer une origine assez reculée pour cette chanson qui aurait été diffusée par des voyageurs de passage au Détroit à l'époque où la région était un véritable carrefour des Pays d'en Haut. Elle représenterait donc une véritable contribution du Détroit au répertoire canadienfrançais.

\section{Conclusion}

On voit par ces quelques échantillons que j'ai pu recueillir que la tradition de « faire des chansons » était présente au Détroit dès la fondation de la colonie et a servi à travers les siècles comme moyen efficace d'exprimer les valeurs de la communauté, de commémorer des événements importants, de maintenir une relation vivante avec la tradition. " Faire des chansons » et les partager avec les membres de sa communauté donne un moyen de participer non seulement au maintien de la tradition, mais aussi à son renouveau.

Alors pourquoi cette tradition semble-t-elle être en perte de vitesse aujourd'hui ? Je crois d'abord qu' un répertoire de chansons traditionnelles d'une certaine profondeur et largement partagé

Détroit. Journaille Commanséle 29 octobre 1765 pour le voiage que je fais au Mis a Mis, Québec, les Presses de l'Université Laval, 2010, p. 107. 
est nécessaire pour la facture de chansons locales. Comme telle, la chanson de composition locale me paraît un marqueur fiable de l'état des traditions orales dans un terrain quelconque. Étant donné que la transmission du répertoire a été interrompue dans cette région, les modèles deviennent de moins en moins familiers. Les formes et les structures que les gens apprenaient autrefois dès leur enfance sont maintenant mal connues et mal comprises ; la poétique traditionnelle, le « prêt-à-porter langagier » et les valeurs qu'ils véhiculent ne font plus partie de la conscience collective. Dans cette région particulière, où la survie de la langue elle- même est en jeu, les références culturelles sont plutôt anglophones sinon carrément états-uniennes. Une chanson calquée sur $L a$ Fille matelot n'est plus la réponse automatique à une expérience vécue. D'ailleurs, le contexte de la performance a changé : les occasions telles que les soirées, noces ou autres rassemblements francophones où les gens chantent ensemble se font de plus en plus rares. Sans doute existe-t-il toujours des individus qui composent des chansons sur des airs connus, de sources et de styles variés, et peut-être même à l'occasion en français. Par exemple, Jean-Pierre Pichette a recueilli une chanson à l'est de Windsor en 1988 qui décrit les efforts déployés par un groupe d'agriculteurs à Pointeaux-Roches pour faire pousser la plus grosse citrouille lors d'un concours annuel dans le cadre du Festival de la Moisson (voir $\mathrm{n}^{\circ} 19$ en annexe) ${ }^{51}$. Mais l'existence et la diffusion de ces pièces semblent plus éphémères que jamais.

Loin de mériter de « mourir dans l'oubli », ce répertoire de chansons locales mérite une place d'honneur dans le patrimoine oral de la région. Il nous en dit long sur le groupe qui l'a produit et transmis, nous révélant des aspects de son histoire cachée ou supprimée, ses attitudes envers des sujets tabous, des descriptions de ses activités quotidiennes et des portraits de personnages qui autrement auraient laissé peu de traces dans la mémoire collective. Linguistes, historiens et ethnologues y trouvent leur compte. On

51. La Citrouille, coll. Jean-Pierre Pichette, enreg. $\mathrm{n}^{\circ} 2867$; chantée par Philippe Charrette, Sainte-Claire-Beach, le 30 mai 1988, Anthologie de littérature orale au Canada français, Sudbury, 1999, p. 234-235. 
peut répéter pour les chansons locales du Détroit ce que Georges Arsenault a dit des chansons acadiennes :

L'étude de la chanson locale présente, à notre avis, un intérêt de tout premier ordre. [...] ces documents oraux sont un apport très important à la petite histoire, voire à l'histoire de la culture acadienne. Ils nous renseignent abondamment sur la mentalité, les mœurs et les préoccupations de ce peuple longtemps ignoré et mal connu ${ }^{52}$.

La disparition de cette tradition suscite une double perte : le monde perd une fenêtre sur une communauté et la communauté elle-même perd un miroir dans lequel elle peut voir reflétées ses valeurs, son histoire, sa vision du monde.

Évidemment, ce processus se perpétue par d'autres moyens. Ce qui se faisait autrefois face à face dans un groupe restreint se fait aujourd'hui via Facebook et YouTube. L'ethnotexte - le « discours qu'une communauté tient sur elle-même, son passé, son présent et son avenir »-s'élabore davantage aujourd'hui dans des formats électroniques. Ces nouvelles modalités entraînent avec elles une redéfinition même du concept du « local». Mais l'idée d'éléments culturels profondément enracinés dans un lieu particulier, évoluant selon des conditions historiques et sociales propres à cet endroit, est parfois difficile à envisager dans un monde branché et de plus en plus sans frontières culturelles. On ne doit pas nier les possibilités de ce nouveau monde, mais cette réflexion sur l'art de " faire des chansons » est peut-être un bon point de départ pour considérer les conséquences de ce grand changement. Il me semble qu'en oubliant l'art de faire des chansons, on se prive d'un élément significatif du discours que les membres d'une communauté tiennent entre eux et avec la tradition qui les unit.

52. Georges Arsenault, Complaintes acadiennes de l'Île-du-Prince-Édouard, Montréal, Les Éditions Léméac, 1980, p. 12. 


\section{Annexe}

\section{Marie Frisine}

Marie Frisine a-t-un nez long,

Pis alle a l'aye $e^{53}$ fait' comme un piton.

Les épaules en gondol',

Avec un grand reinquier,

Les jamb's comm' l'alouette,

Les pieds comme des morquiers ${ }^{54}$.

Son mari arrive du champ,

[I'voudrait bien de quoi manger.]

- Mais, dis-moé donc, Frisin',

Quoi tu fais aujourd'hui?

- J'ai faite des nouvelles

Toute l'avant-midi.

Marie Frisine est jamais démontée,

Mais alle a eu des crêpes à démêler.

[Des crêpes à démêler.]

Ça prendrait pas grand temps.

Pis a' ménag'rait la graisse

Pour tous les p'tits enfants.

Marie, veux-tu [a]ttend' un p'tit moument,

Un habit d'plum's $m$ 'as te donner ${ }^{55}$.

Lavé dans une teinture,

Et aussi repassé,

Ça s'ra pour la plus belle

De toute la compagnée.

Marie Frisine, coll. Marcel Bénéteau 603.33a ; chantée par Gilbert LaVoy, le 17 octobre 1942 à Deerfield, Michigan ; enregistrée sur disque vinyle par la famille Réaume/ LaVoy ; source : Dennis Au. Titre du Catalogue Laforte : Marie Frisine (II. O-90).

53. L'œil [peut-être « elle a l'air faite comme un piton »?

54. Mortiers.

55. Je m'en vais te donner. 


\section{Quand c'que nous sommes dans ces prisons}

Quand c'que nous sommes dans ces prisons, / tou[s] prisonniers

ensemble,

Tou[s] prisonniers, tou[s] bons lurons, / tou[s] de la même bande.

Nous passions le rest' de la nuit

Entre les bras de nos Clari[s].

Et quand c'qu'i[1s] nous mett'nt dans un cachot / enteur ${ }^{56}$

quatre murailles,

Rongés des poux, rongés des rats, / couchés dessur la paille.

Grand Dieu qu'ont ces tristes méquiers ${ }^{57}$,

Qu'ont donc ces pauvres prisonniers.

Quand c'qu'i[ils] nous sort'nt de la prison / pour aller nous fair'

pendre,

I' nous mett'nt les ménottes aux pieds, / c'est pour que nos

cœurs grondent.

Grand Dieu qu'ont ces tristes méquiers,

$\mathrm{Qu}$ 'ont donc ces pauvres prisonniers.

Quand ce que nous sommes dans ces prisons; coll. Marcel Bénéteau 501.14a ; chantée par Stella Meloche, le 14 avril 1993, Petite-Côte, Rivière-aux-Canards ; chanson de son père, Henri Meloche. Titre du Catalogue Laforte : Les Prisonniers politiques (VI. B-55)

\section{Les Prisonniers politiques}

Il nous faut faire une chanson

Tous prisonniers ensemble

\} bis

Il nous faut faire une chanson

Puisque nous sommes dans la prison.

Quand tu me parles de chanter

Cher ami tu m'affliges

Il nous vaudrait bien mieux pleurer

Que dedans la prison chanter.

Quand nous veillions chacun chez nous

Le soir à la chandelle

56. « entre », par métathèse.

57. Métiers. 
Nous étions heureux et contents,

Nous vidions les bouteilles

Passant la nuit

Au côté de sa chère amie.

Musée National, coll. É.-Z. Massicotte, ms nº 915, 1922. Titre du Catalogue Laforte : Les Prisonniers politiques (VI. B-55).

\section{Les Prisonniers}

- Nous faut chanter une chanson, / tous prisonniers ensemble. (bis) Tous prisonniers, tous beaux garçons / des lurons à la danse.

Nous faut chanter une chanson / puisque nous sommes dans la prison. $\}$ bis

- Quand tu me parles de chanter, / camarade, tu m'affliges, Comment veux-tu que je chante en prison / avec le cœur si triste, J'aurais plutôt le regret de pleurer / que dans la prison chanter.

Mais quand y vient le lun ${ }^{58}$ matin / nous interroger sans doute, Les fers aux pieds, les mains liées, / grand Dieu ! quelle souffrance, C'est pour nous faire déclarer / les crimes que nous n'avons pas faits.

Le meilleur de notre repas, / c'est un peu de potage, C'est pour nous garantir le trépas / lorsque nous sommes mis en cage, Oh ! voilà le triste métier / que font les pauvres prisonniers.

Voilà si bien comment se réduit / entre quatre murailles, Piqués des poux et des souris / et couchés sur la paille, Oh ! voilà le triste métier / que font ces pauvres prisonniers.

- Mon bel ami, te souviens-tu pas / quand nous vidions la bouteille ! Combien la vidions si souvent / les soirs à la chandelle, Passant le restant de la nuit / entre les bras de nos chères fleuries.

Les Prisonniers, recueillie en 1871 par Victor Smith ms 6848, f. 186-187 ; chantée par Nannette Lévesque. Catalogue [Répertoire] Coirault : 6224, Prisonniers pour des crimes qu'ils n'ont pas faits. Publié dans Georges Delarue et Marie-Louise Tenèze (dir.) Nanette Lévesque conteuse et chanteuse, Paris, Éditions Gallimard, 2000, p. 615-616.

58. Lundi. 


\section{Les Filles de Sandwich}

C'est dans Sandwich, le petit faubourg,

[II] y a des fill's tout alentour.

Il y en a des petit's pis des grandes

Qu'ils veulent s'y marier,

Mais personn' les demande.

Tou[te]s les fill's sont rassemblées,

Y a une lett' qu'i[ls] ont composée.

Ils l'emporta le dimanche à la messe :

- Tenez monsieur l'curé,

Publiez cettre lettre.

Monsieur l'curé l'a pas manqué,

Après la messe a publié :

- Tous les garçons, les jeun's gens du village,

Oh prenez donc ces filles,

$I l[\mathrm{~s}]$ ont de bon courage.

Tous les garçons n'ont pas manqué,

Après la mess' sont rassemblés :

- Buvons, chantons, caressons la bouteille,

Nous sommes à marier,

Les filles nous appellent.

Les Filles de Sandwich, coll. Marcel Bénéteau 301.02a ; chantée par Cécile Meloche, le 10 février 1990, McGregor. Titre du Catalogue Laforte : Les filles demandent les garçons (II. O-21). 


\section{Les Gens de Sainte-Claire}

C'est les gens de Sainte-Claire

Ce sont tous des gens fiers

Les grands comme les petits

Ils boivent tous du whisky

S'ils n'ont pas ménagé

Pendant leur été

Ils auront de la misère

Pour passer cet hiver.

C'est dans Loiselleville

Que nous sommes tous hivernés

Nous sommes une bonne gang

Et bien fort cassés.

Car les mortifiés,

J'ose pas les nommer ;

C'est Arsène Bondy

Qui est le plus cassé.

C'est Arsène Bondy,

Vous le connaissez bien,

C'est le plus grand débauché,

Le monde font rien qu'en parler ;

Les femmes du village

Sont fâchées contre lui

Elles disent que c'est lui

Qui débauche leurs maris.
C'est chez monsieur Vigneux

Que nous sommes tous invités

Nous sommes invités

Pour aller visiter.

S'ils n'ont pas d'argent

Pour passer le jour de l'An

Ils auront du whisky

Pour traiter leurs amis.

S'ils n'ont pas de whisky

Pour traiter leurs amis

Ils iront en chercher

Où ils l'auront à crédit.

Verse, verse

Une chopine de whisky

$\mathrm{Si}$ on fait une bonne semaine

On vous paiera samedi.

Excusez la chanson

Que je viens de vous chanter

Je l'ai composée

Pour me désennuyer

Je l'ai composée

Sur l'air de Sainte-Claire

Pour mieux en prendre goût

Il faut prendre un petit coup.

Les Gens de Sainte-Claire, coll. Marcel Bénéteau 102.08a ; chantée par Mabel et Maguerite Bondy, 1988, Petite-Côte, Rivière-aux-Canards ; titre du Catalogue Laforte :

Les Tailleurs de pierre (II. O-106).

\section{Les Mascoutens}

Un sauvage chassant dans ces bois, Ayant faim de manger du pain,

Dessur un Français il s'est en allé, Tout épouvanté, disant : - Sauve-toé.

Il lui a dans ces bois beaucoup d'Iroquois 
Qui vont mettre à $y \hat{a}-y o r^{59}$ les Français.

Bourdignon qui est un homm' sans façon, I[1] dit : - Camarades allons.

Tout en continuant vers le commandant, Z-il s'est en allé, disant :- Monsieur Roy, Faites rassembler tous vos garnadiers, Un homm' pour interprèter.

Aussitôt qu'le command'ment fut donné, Tout à chaquin fut rassemblé.

Et les sauvag's contents avec leur butin, Ils s'en allaient tous, i' criaient: - Ya oin !

Oh les Mascoutens patago malins, Tous chargés de ce bon butin.

Les Mascoutens, coll. Marcel Bénéteau 501.21a ; chantée par Stella Meloche le 25 septembre 1993, Petite-Cote, Rivière-aux-Canards; chanson de son père Henri Meloche et de son grand-père Étienne Meloche. Titre du Catalogue Laforte : non répertorié.

\section{Maudit Lincoln}

Sont les republicains / qu'ont électé Lincoln-e.

Quant' i' ont [en]tendu / de mett' les nèg[r]es free,

Les auréol's ont été finies.

La yerre est déclarée, / nous gagnons trois batailles.

C'est à Bull Run / que le feu s'est donné.

Les republicains, ils s'sont sauvés.

Oui mais ils l'ont r-étendu, / étendu sur la terre.

Maudit Lincoln, / tu mérites l'enfer,

Fair' mourir les blancs pour les noirs.

Tu fais d'la fausse argent, / tu la pass' pour d'la bonne.

Si tu le fais, / c'est pour tricher le monde

De cœur en t'plaçant de l'escompte.

59. Imitation du parler amérindien : « I[1s] voulaient dire qu’i étaient pour les jeter dehors. " 
Qui n'a composé la chanson, / je vous quitte savoir-e,

C'est Hubert Clos, / qui l'a faite et composée,

Les démocrat's vont la chanter.

Maudit Lincoln, coll. Marcel Bénéteau 604.12a ; chantée par Moïse et Emma Drouillard, Windsor, le 11 juin 1970 ; enregistré par leur fils, Ted Drouillard. Titre du Catalogue Laforte : non répertorié.

\section{Déménagement de l'école Robillard}

Écoutez, je vas vous chanter

La chanson $d u$ rébellion.

Ils ont été chez Pierre Perreault

Lui dire qu'il vienne avec ses rouleaux.

Mais c'pendant,

Ils croient d'avoir leur deux cents.

Le vieux Papineau qui est bon ouvrier,

Il dit que l'école peut s'mouver.

Jina, qui est indépendant,

Dit qu'elle se mouvera en peu de temps.

Mais c'pendant,

Monsieur Papineau fournira son temps.

Revenant, mais à Poisson,

C'est lui qui pose les fondations.

Il dit : - Faut se mettre en sûreté,

Pour pas qu'elle écrase dans les écoulés,

Mais c'pendant,

Monsieur Papineau fournira son temps.

Guillaume Ladouceur,

Qu'est bon empereur.

Il dit : - Faut sacrifier notre cœur,

Il faut aller voir Paul Sauvé.

Mais c'pendant,

Monsieur Papineau fournira son temps. 
Faut aller voir Crapaud Girard, Pour voir s'il va fournir sa part.

- Vous n'aurez qu'à m'avertir, Ah oui, j'irai d'un grand plaisir.

Mais c'pendant, Monsieur Papineau fournira son temps.

Faut aller voir Jos Girard Pour voir s'il va fournir sa part. Faut aller chercher Bébé Meunier Pour nous aider à la traîner.

Mais c'pendant, Monsieur Papineau fournira son temps.

Quand ils se sont fait prêcher

Le jour de Noël, ça les a fait diabler.

Garçon Girard sort dehors, Il dit : - Ça parle au diable !

Mais c'pendant, Monsieur Papineau fournira son temps.

Faut avoir le vieux Beauchesne, Pour nous accrocher les chaînes. Faut aller chercher Paul Diesbourg Pour nous aider à la sortir d'la cour.

Mais c'pendant, Monsieur Papineau fournira son temps.

Revenant à Jos Dupuis, Il s'en est mélé aussi.

Liboire, qui n'a pas grand affaire,

Mais il leur dit de tenir leur tête.

Mais c'pendant, Monsieur Papineau fournira son temps.

Faut aller le vieux B. Sauvé, Pour nous conduire et nous viser.

- Comment voulez-vous que j'aille,

Chers amis ? J'ai rien qu'un oeil.

Mais c'pendant, Monsieur Papineau fournira son temps. 
Le vieux Papineau, tout égriché,

Il faut l'avoir pour nous crier.

Il nous crira «Ah ji, ah ha!»

Breffement pour commander ça.

Mais c'pendant,

Monsieur Papineau fournira son temps.

- Moïse Papineau, si t'es électé,

On dit tu seras le trésorier.

Il dit : - Si je gagne mon élection,

Je ne mouverai pas la maison.

Mais c'pendant,

Monsieur Papineau fournira son temps.

Revenant à Colombe Granger,

Il dit qu'il a une grosse idée.

Il dit que rien l'embarrasse,

On va le mettre à écorner les vaches.

Mais c'pendant,

Monsieur Papineau fournira son temps.

Revenant à Johnny Papineau,

Sa grosse face noire de crapaud.

Ils ont été pour la faire signer,

Mais il lui en a bien empêché.

Mais c'pendant,

Il lui a montré les grosses dents.

Sa mère, elle lui a dit :

- Tu n'es pas le maître ici.

Oh, tu fais trop l'indépendant,

Tu pourras saprer ton camp.

Mais c'pendant,

Johnny Papineau a fourni son temps.

Revenir à la misère, mais que ces gens

Ça aillent à [la] confesse.

Moïse dit qu'il s'en occupe pas,

Il y va pas tous les mois.

Mais c'pendant,

Il dit qu'ils gardent leurs sacrements. 
Jina, c'est lui qui est l'plus effronté,

Il a été se présenter.

Ils ont pas voulu le recevoir,

Ils l'ont envoyé au presbytère.

Mais c'pendant,

Il a pas eu les sacrements.

Revenir au mardi soir,

C'est quand ils ont fait leur fête.

Ils étaient comme à la guerre,

C'était d'avoir trop bu d'bière.

Mais c'pendant,

Ils ont répandu leur sang.

Bouteillé qui est conseiller,

Pour conduire les têtes de boulé.

Moïse Lambert qui est à la tête,

C'est lui qui est la plus grosse bête.

Mais c'pendant,

Monsieur Papineau fournira son temps.

Qui en a composé la chanson,

C'est Pierre Jervais et son garçon.

En revenant de la Pointe-aux-Roches,

Assis sur un voyage de roches.

Mais c'pendant,

Monsieur Papineau a fourni son temps.

Déménagement de l'école Robillard, coll. Marcel Bénéteau 604.20a ; manuscrit de Léo Plante, Belle-Rivière, le 23 juillet 1930, p. 23-28. Titre du Catalogue Laforte : non répertorié.

\section{Le Pier de Belle-Rivière}

En passant par Belle Rivière,

J'ai arrêté chez Gauthier pour la bière.

Chaque côté de moi j'ai vu

Des visages inconnus

Et qui parlaient tous du pier.

Un dit que dans cinq ans ça sera pourri

Avec tout l'argent que le gouvernement a fourni. 
Et l'autre dit que cet argent est perdu ;

Mais il se trompe elle est dans la poche de $\mathrm{McCu}$.

Cependant, il y a le père Charles

Qui est le bosse et un fin marle.

Tous les mois il reçoit un chèque

Avec $\$ 70.00$ en se plissant le bec

En disant : - Il en faut un à la Rivière à Peck.

Il s'approche de $\mathrm{McCu}$ en disant :

- Il faut se taire ou nous sommes foutus,

Car ce pier n'est pas fait par archetèque,

On perdra celui de la Rivière à Peck.

Le Pier de Belle-Rivière, coll. Marcel Bénéteau 604.29a, publiée dans Le Progrès, le 2 octobre 1884, p. 2.

\section{Défaite de McKee à Belle-Rivière}

Écoutez, je vas vous chanter

Une chanson de vérité.

C'est du Dr. Reaume

Qui a eu la majorité.

Celui qui a eu du chagrin

C'est ce pauvre Sévérin, Après avoir parlé si fort

Et avoir resté dehors.

Parlons donc de Baptiste Sauvé,

Après avoir couru les Très-Carrés,

Il a couru si fort

Qu'il a trouvé Tit Charles Rivard.

Quand Charles vint pour voter

Il a regardé de côté.

Pour se réjouir le cœur,

Il a voté Conservateur.

À revenir au gros Girard, Lui qui avait l'air d'un si gros petard,

Il a pété si fort

Que Billy a été jeté dehors. 
Parlons donc de Louis Désilet,

Lui qui avait fait son emplette.

Je vous assure qu'il a bien fallu

Qu'il emplise sa tinette.

Celui qui ne voulait pas Réaume

C'est ce pauvre Fred St-Onge.

Il a bien faullu qu'il prenne la queue

Comme ce pauvre écornifleux.

Ce qui n'est point de valeur

C'est de rencontrer les Conservateurs.

Ils ont le cœur très gai

Parce qu'ils ont balayé Billy.

Ils ont balayé si fort

Que Billy $a$ resté dehors.

Celui qui n'était pas trop content

C'est ce pauvre Charles Adam.

Celui qui n'a pas ri comme il faut, C'est Honoré Trotechaud.

Celui qui n'avait pas de façons,

C'est ce pauvre Hormidas Caron.

Je vas terminer la chanson

Sur ce bon rouge comme Peter Dumont.

Il a été malade bien fort

Parce que Billy a resté dehors.

Défaite de McKee à Belle-Rivière, coll. Marcel Bénéteau 604.31a ; publiée dans Le Progrès, le 3 juillet 1902, p. 2. Titre du Catalogue Laforte : non répertorié. 


\section{Défaite de McKee à Belle-Rivière}

C'est dans l'année dix-neuf cent deux,

Une élection avait eu lieu

Entre libéraux et conservateurs :

Les deux partis ont eu bien peur,

Mais McKee avait bien l'idée

Que ce serait lui qui serait entr[é].

Quand les retours sont arrivés

Les libéraux se sont dispersés,

Quand la déclaration fut fait[e] Mon pauvre McKee était défait. Mais malgré tou[s] ses efforts, Il a fallut coucher dehors.

Les libéraux ont pour défense Que Réaume a fait des dépenses. C'est difficile de douter

Les deux partis ont travaillé. S'il y a eu des reçus de donné[s], Je crois que McKee les a signé[s].
J'aurais voulu voir Casgrain

Afin de lui toucher la main Et lui dire d'affiler son couteau, Pour McKee lui lever la peau, Afin de la faire sécher Pour ne jamais se présenter.

Tous mes amis les électeurs, Ne trouvez pas cela de valeur. Il y a bien assez longtemps Qu'il n'a pas eu de changements, Aujourd'hui on s'est décidé Que le parti allait changer.

Pour terminer ma chanson, Qu'on m'apporte verre et flacon, Que je boive à la santé De celui qui est entré. J'espère qu'il fera son devoir Comme il l'a promis l'autre soir.

Défaite de McKee à Tecumseh, coll. Marcel Bénéteau 604.30a ; publiée dans Le Progrès, le 5 juin 1902, p. 1. Titre du Catalogue Laforte : non répertorié.

\section{La Vendeuse d'œufs}

C'est une vendeuse d'œufs

De notre paroisse.

Elle avait pris ce commerce,

C'était comme une bourgeoise.

Elle allait tous les jours en ville, Toujours avec son panier vide. Encore qu'elle était traversée, Son panier était encomblé. 
Si vous connaissez pas le soupçon,

Je vas vous conter l'affaire :

Elle les mettait dans son jupon

Sans que personne les puisse voir.

Elle les traversait par douzaines,

Mais toujours sans payer douennes.

Les officiers l'ont remarquée,

Ils la trouvaient si drôle.

Ils l'ont faite rentrer dans l'office,

Croyant d'y faire gros bénéfice.

Les œufs ont tous cassé,

La bonne dame s'est en allée.

Les officiers l'ont rappelée

Pour y faire nettoyer son dégobillage.

La bonne dame s'est point souciée,

Elle mena le carnage.

Elle les traita d'effrontés,

D'empêcher les femmes de smuggler.

Elle leur a dit leurs vérités,

Après s'est en allée.

La Vendeuse d'œufs, coll. Marcel Bénéteau 604.28a, mansucrit d'Edwidge Monforton, Ojibway (Windsor), 1908, n 191 . Titre du Catalogue Laforte: non répertorié.

\section{4. Écoutez, je m'en vais vous chanter}

Écoutez, je m'en vais vous chanter

Une chanson que j'ai composée.

Vous allez peut-être dire,

Vous allez aussi en rire.

Elle a été faite assurément

Sur un de nos bons habitants.

Vous connaissez tous monsieur Prieur,

On dit que c'est un homme de cœur.

Il a $v u$ assez de courage

De tenir sa femme dans la cave.

Elle a tant coupé de queues,

Qu'elle [e]n a perdu les deux yeux. 
On dit qu'il est bien malheureux

Depuis sa femme a perdu les yeux.

Il l'a tant tenu dans la cave

À couper des queues de bétteraves,

À couper des queues de navots,

Pour soigner ses animaux.

M'en revenant de faire mon tour,

J'ai rentré sur Saint-Amour.

J'ai bien vu au ras la porte,

Prieur qui se marchandait des bottes.

- J'ai un marché à vous proposer,

Si vous voulez l'accepter.

Saint-Amour se tenant autour

Et voulut savoir le tour.

Il dit : - J'ai cent minots de carottes

Que je vous donnerai pour vos bottes.

Si vos bottes ne pren[nent] pas l'eau,

Je vous donnerai dix minots de navots.

Saint-Amour, restant surpris,

Dit : - Monsieur Prieur, vous n'avez pas d'esprit.

Ne me prenez pas pour un sot,

De vous, des bottes pour des carottes.

Allez porter vos carottes au marché

Et ensuite vous viendrez me payer.

L'autre jour, je me rappelle,

Deux dames qu'ont été acheter du miel.

Ils l'ont mis dans un si grand transport,

Il dit : - Moi, je m'en vas dehors.

Je ne peux plus les envisager,

Car tout mon miel ils vont manger.

Ces deux dames $s$ 'entregardaient,

Vous pouvez penser que $a^{\prime}$ ' [elles] riaient.

Il y en a une qui dit à l'autre :

- Moi, je m'en fous, ce n'est pas de ma faute.

C'est toi qui porte[s] le plus gros fardeau,

C'est toi qu'a[s] mangé gâteau.

Je me souviens de temps passés, 
Il était toujours en geste,

Quand il rôdait avec sa tresse.

Quand il tressait pas, il tricotait,

Ses hommes au bois, qu'ils bûchaient.

Je me souviens encore très bien,

Quand il a pris madame Chrétien.

Quand il a acheté sa terre,

Il n'avait pas son contrat clair.

Mais elle a donné son contrat

Pour faire chanter son Libéra.

En revenant de l'écurie,

Prieur n'était endormi.

Il vient se mettre à la porte,

Avec son air épouvantable,

En criant : - Jézan d'un bleu,

Poupa, avez-vous soigné bœu[f] [?].

Qu'en a composé la chanson,

C'est Pageau, ce bon garçon,

Qu'il dit en tout temps et en toute heure,

Qu'il n'a pas peur de monsieur Prieur,

Qu'il pourra lui chanter

Quand il voudra l'écouter.

Écoutez, je m'en vais vous chanter, coll. Marcel Bénéteau 604.23a, manuscrit de Félix Drouillard, Rivière-aux-Canards, vers 1897-1903, p. 90-92. Titre du Catalogue Laforte : non répertorié.

\section{Le Vieux Baptissette Bebi}

Du long de la rivière, / su[u] l'vieux Baptisett' Bebi,

I[1] a eu un' triste chose / qui s'trouvait l'lundi.

I[1s] ont fait un' courvée,

C'était pour acc[o]mmoder,

Ils ont fait des bêtises, / ça leur sera payé.

Le long de la veillée, / quosqu'on a vu entrer?

Le vieux Bat[is]sett' Bebi, / qui était à moitié g'lé.

- Tu sais, ma Joséphine,

Qu'on s'est toujours promis

Qu'i[1] aura jamais de danses / ou de veillées ici. 
C'était madam' Bapt[is]sett', / le nez tout picoté,

Les cheveux dans la face, / comme un' bottine de blé.

- Eh bien, madam' Bapt[is]sett',

Vous 'n avez fait assez,

Donnez-nous tout's nos cas[ques], / nous allons tous s'en aller.

- Tu sais, ma Joséphine, / que j't'ai jamais battue, M'en vas t'en donner un' / comm't'en as jamais eu.

On entendait les coups,

Du curé chez Mailloux,

Ell' criait comme un' bête / qui s'faisait casser l'cou.

Du long de la semain', / du long du Trécarré,

Tout le mond' nous demande : / - Ivou c'que vous d'venez ?

- On vient d'su[r] l'vieux Bapt[is]sett',

On vient de la courvée,

Nous ont fait des bêtises, / ça leur sera payé.

Le Vieux Baptissette Bebi, coll. Marcel Bénéteau 304.07a ; chantée par Ubald (Slim) Ducharme le 6 avril 1993, LaSalle, né à Tecumseh ; chanson de son père, Herbie Ducharme. Titre du Catalogue Laforte : non répertorié.

\section{La Chanson du Détroite}

C'est dans le bord du Détroite, / près d'au d'sus de transvoyé.

Où c'qu'il y a de jolies filles / qui veulent se marier.

Les amants qui vont les voir, / z'ils ont beaucoup d'amitié.

Lisette, en faisant la fière / a bien su les renvoyer.

- Fais pas tant la difficile. / Si tu veux y avoir mon cœur, Ce n'est pas par tes manières / ni tes façons de grandeur. Les amants que tu refuses, / tu les r[e]gretteras un jour. Tu resteras vieille fille, / date de tes tendres amitiés.

C'est dans le bord du Détroite / qu'on a eu tant d'agrément. On jouait au jeu de cartes, / c'était tout notre passe-temps. [On ?]' ah ! ma charmante bouteille / qui me cause tant de tourment ! Je vous jure dessur mon âme, / quand je bois je vis content.

La Chanson du Détroite, coll. Marius Barbeau n 1962, juin 1919, Musée National, chantée par Ovide Soucy. Titre du Catalogue Laforte : non répertorié. 


\section{Tout autour de ces îlettes}

Tout autour de ces îlettes, Dans des souterrains noyés. Il y a des jolies fillettes, Qui sont bonnes à marier. Les amants qui vont les voir Avec beaucoup d'amitié, Ma Lisett' qui fait sa fière A bien su les renvoyer.

« Lisett', fais pas tant la fière Si tu veux avoir mon cœur. $\mathrm{Tu}$ connais pas la manière, Ni la façon de mon cœur. Les amants que tu refuses Viendront un jour à manquer. Tu t'en trouveras la dupe Avec tout's tes grand's fiertés.

« Adieu, les fill's du village, Où j'ai eu tant d'agrément. Le jeu de dés et de cartes, C'est là tout mon agrément. C'est cett' charmante bouteille Qui me cause tant de tourments. Ah! j'vous jure en honnête homme, Plus je bois, plus j'vis content. »

Tout autour de ces îlettes, coll. É.-Z. Massicotte, $\mathrm{n}^{\circ}$ 779, 1919, Musée National ; chantée par Vincent-Ferrier de Repentigny. Publiée dans Charles-Marius Barbeau, Le roi boit. Troisième partie du Répertoire de la chanson folklorique française au Canada, Lucien Ouellet, éditeur, Ottawa, Musée canadien des civilisations, Musées nationaux du Canada, 1987, p. 83-84. Titre du Catalogue Laforte : non répertorié.

\section{Adieu donc, belle rivière}

C'est au bord de la rivière,

Chez monsieur, vous le savez,

Il lui a des jolies filles

Qui voudraient s'y marier. 
Les amants qui vont les voir,

C'est pas par amitié ;

Marianne par son air sévère

Les a bêteau [bientôt] renvoyés.

Marianne fais pas tant ta fière

Si tu veux avoir mon cœur.

Je n'aime pas tes manières,

$\mathrm{Ni}$ tes façons de grandeur.

Les amants que tu rebutes,

Un jour ils te manqueront;

Tu t'en trouveras la dupe,

Marianne ne le crois-tu pas?

Adieu donc, belle rivière

Où j'ai eu tant d'agrément,

Où j'ai tant joué aux cartes,

C'était tout mon élément.

Ah, c'est ma chère bouteille

Qui m'a causé tout cela;

Je vous jure en honnête homme,

Celui qui $d o i t^{60}$ ne vit pas.

Adieu donc, belle rivière, coll. Marcel Bénéteau 204.19a ; manuscrit de Félix Drouillard, Rivière-aux-Canards, vers 1897-1903, p. 134. Titre du Catalogue Laforte : non répertorié.

\section{La Citrouille}

Un bon soir, à la fin d'octobre,

Les gars on dit aux femmes d'aller mettre leurs robes.

Ils sont allés au Festival de la moisson

Là, pour voir, des gars, qui était le plus bon.

La citrouille, oh! la citrouille,

La plus gross' peut causer la gribouille.

Notre Ursule a été bien claire,

Elle a dit aux gars exactement quoi faire :

60. Sans doute « boit », comme dans la version chantée par Stella Meloche : «Celui qui ne boit vit pas » (coll. Marcel Bénéteau 204.19b). 
Avant de la peser, ne pas briser la tige,

Me d'mandant, ce foyer, c'est qui qui dirige.

Le premier à se déclarer,

C'était pas un Chauvin qui voulait se vanter.

Il avait assez hât' de se rendre chez lui

Qu'il a eu trois amendes de policiers.

Paul Lebeuf n'a pas été plus fin,

Il a oublié de bien guetter son chien ;

Quand il est sorti, il a le cœur tout gros :

Sa gross' citrouille était en p'tits morceaux.

Qu'est-c' qu'on peut dir' de Rosair' Sylvestre, Il était plus illuminé que l'reste ; Au-d'ssus de sa plante, il a posé un' lumière ;

Dieu aura voulu qu'il dise ses prières.

Pour être rusé et gagné le « bet», Il a mis sa plant' dans une grand' cachette ;

Quand est venu le temps de la peser, René Sylvestr' ne pouvait plus la trouver.

Faut pas oublier le [Hou] Grondin Même si leur citrouille n'aboutissait à rien ; Ell' n'était pas grosse, j'ai entendu dire, C'était des citrouilles à nous fair' rire.

François Caron ne pouvait pas se tenir : Il allait gagner, cela allait sans dire ;

Cette attitud' lui a causé de la brouille :

Tous les gars ont dit qu'il était plein de citrouilles.

Si jamais je vais au paradis, Je demanderai s'il y a des citrouilles ici ; Si saint Pierre me dit que " oui, il y en a », Je vais demander d'être envoyé en bas.

La Citrouille, collection Jean-Pierre Pichette, enreg. no 2867 ; chantée par Philippe Charrette, Sainte-Claire Beach, le 30 mai 1988. Titre du Catalogue Laforte : non répertorié. 